\title{
Quantized Consensus ADMM for Multi-Agent Distributed Optimization
}

\author{
Shengyu Zhu, Mingyi Hong, and Biao Chen
}

\begin{abstract}
Multi-agent distributed optimization over a network minimizes a global objective formed by a sum of local convex functions using only local computation and communication. We develop and analyze a quantized distributed algorithm based on the alternating direction method of multipliers (ADMM) when inter-agent communications are subject to finite capacity and other practical constraints. While existing quantized ADMM approaches only work for quadratic local objectives, the proposed algorithm can deal with more general objective functions (possibly non-smooth) including the LASSO. Under certain convexity assumptions, our algorithm converges to a consensus within $\log _{1+\eta} \Omega$ iterations, where $\eta>0$ depends on the local objectives and the network topology, and $\Omega$ is a polynomial determined by the quantization resolution, the distance between initial and optimal variable values, the local objective functions and the network topology. A tight upper bound on the consensus error is also obtained which does not depend on the size of the network.
\end{abstract}

\section{Index Terms}

Multi-agent distributed optimization, quantization, alternating direction method of multipliers (ADMM), linear convergence.

\section{INTRODUCTION}

$\mathbf{T}$

HERE has been much research interest in distributed optimization due to recent advances in networked multiagent systems [1], [2]. For example, ad hoc network applications may require agents to reach a consensus on the average of their measurements [3], including distributed coordination of mobile autonomous agents [4], and distributed data fusion in sensor networks [5]. Another example is the large scale machine learning where a computation task may be executed by collaborative microprocessors with individual memories and storage spaces 
[6], [7]. Many of the distributed optimization problems, such as those mentioned, can be cast as an optimization problem of the following form

$$
\min _{\tilde{x}} \sum_{i=1}^{N} f_{i}(\tilde{x}),
$$

where $f_{i}: \mathbb{R}^{M} \rightarrow \mathbb{R} \cup\{\infty\}$ is the local objective function associated with agent $i$. The function $f_{i}$ is composed of a smooth component $g_{i}: \mathbb{R}^{M} \rightarrow \mathbb{R} \cup\{\infty\}$ and a non-smooth component $h_{i}: \mathbb{R}^{M} \rightarrow \mathbb{R} \cup\{\infty\}$, i.e., $f_{i}=g_{i}+h_{i}$. Examples of such models include least squares [8], [9] and regularized least squares [10]-[12]. The variable $\tilde{x}$ may represent average temperature of a room [5], frequency-domain occupancy of spectra [12], states of smart grid systems [13], etc.

In the above scenarios, it is commonly assumed that each agent only has the knowledge of its local objective function, and a fusion center is either disallowed or not economical. As such, the agents seek to solve (1) collaboratively using only local computation and communication. In practice, a number of factors, such as limited bandwidth, sensor battery power, and computing resources, place tight constraints on the rate and form of information exchange amongst neighboring agents, resulting in the quantized communication constraint. The challenge of this paper is to obtain, for each agent, a reasonable solution to (1) in a distributed manner under the quantization constraint.

Existing methods that handle this constraint include quantized incremental algorithm [14], quantized dual averaging [15], and quantized subgradient method [16]. The quantized incremental algorithm achieves a worst case error which is roughly $O(1 / \sqrt{k})$, where $k$ is the number of iterations, for specific quantization resolutions. This algorithm, however, does not guarantee to reach a consensus nor to converge as $k \rightarrow \infty$. The quantized dual averaging method reaches a neighborhood of the optimal solution at a rate of $O(1 / \sqrt{k})$, but also does not ensure the convergence or a consensus. The quantized subgradient method converges to a consensus within a neighborhood of the optimal solution at a rate of $O(1 / k)$, whose consensus error, i.e., the difference between the convergent value and the optimal value, increases in the quantization resolution, the size of the network, and the largest norm of the subgradients of local objective functions. To the best of our knowledge, there are no accelerated rates established for these algorithms when local objective functions are further known to be strongly convex.

It is important to note that existing quantized algorithms all have sublinear rates to reach a neighborhood of the optimal solution. This is because their respective standard versions, i.e, the incremental algorithm [17], the dual averaging method [18], and the distributed subgradient descent algorithm [19], have slow convergences. In addition, the errors of these quantized algorithms from the optimum tend to increase when the network becomes larger, which is much undesired as large scale networks are very typical in today's applications. The alternating direction method of multipliers (ADMM) has been known as an efficient algorithm for large scale optimizations 
and used in various applications such as regression and classification [20]. It has been shown to have a sublinear convergence rate $O(1 / k)$ for general convex optimization problems [21], and to be linearly convergent for certain objective functions [22], [23]. Recent work of [24] also extends the linear convergence to a distributed ADMM method using synchronous steps when the local objective functions are strongly convex and have Lipschitz gradients. We hence expect an ADMM based quantized algorithm working well for solving (1) in terms of both the consensus error and the convergence time.

Unfortunately, when the quantization constraint is imposed, existing ADMM methods can only deal with quadratic local objective functions [8], [9]. With dithered quantization [25], using the facts that quadratic functions have linear gradients and that the expectation of the dithered quantizer output is equal to the input, one can show that each agent variable converges to the optimal solution in the mean sense. For deterministic quantization, the idea is to rewrite the update as the sum a standard ADMM update of the agent variables plus an accumulated error term caused by quantization, and then use the linear convergence rate to establish convergence. These approaches, however, do not apply to general convex objective functions (see also [9, Remark 6]). The local objectives can be non-smooth (e.g., the LASSO). Even when they are smooth, their gradients are not necessarily linear. Therefore, the effect of dithered quantization is hard to characterize and one can hardly write out the quantized update as the sum of a standard ADMM update plus an accumulated error term. Moreover, the linear convergence rate of the standard ADMM might fail to hold, making it more difficult to deal with quantization.

Our main contribution is to develop a quantized distributed ADMM algorithm using deterministic quantization. We do not directly prove the convergence of the variables at each agent; instead, we seek to establish the convergence of an auxiliary vector which determines the update of the agent variables. In particular, we show that this algorithm converges to a consensus within finite iterations under certain convexity assumptions as long as an initialization condition is satisfied. The initialization condition is rather mild; indeed, simply setting all the variables to 0 suffices. We derive a tight upper bound on the consensus error which does not depend on the size of the network. We finally characterize the convergence time, that is, our algorithm converges within $\log _{1+\eta} \Omega$ iterations where $\eta>0$ depends on the local objectives and the network topology, and $\Omega$ is a polynomial decided by the quantization resolution, the initial variable values, the local objective functions and the network topology. The proof idea also provides a framework for convergence proof of other quantized algorithms (see Section 世II-B).

The rest of this paper is organized as follows. Section $\amalg$ reviews the application of the ADMM to distributed optimization without the quantization constraint, resulting in a distributed ADMM algorithm. In Section we use deterministic quantization to modify the above algorithm to handle the quantization constraint. We show the relation of the quantized algorithm to the standard ADMM and establish the desired convergence results. Simulations are provided in Section IV] followed by conclusion in Section $\mathrm{V}$ along with discussions on future research directions. 
Notations: We use 0 to denote the all-zero column vector with a suitably defined dimension. $1_{K}$ is the $K$ dimensional all-one column vector; $0_{K}$ and $I_{K}$ are the $K \times K$ all-zero and identity matrix, respectively. Notation $\otimes$ denotes the Kronecker product and $\|x\|_{2}$ denotes the Euclidean norm of a vector $x$. Given a positive semidefinite matrix $G$ with proper dimensions, the $G$-norm of $x$ is $\|x\|_{G}=\sqrt{x^{T} G x}$. Denote $\sigma_{\max }(D)$ as the largest singular value of a matrix $D$ and $\tilde{\sigma}_{\min }(D)$ as the smallest nonzero singular value of $D$. $\partial f(x)$ denotes a subgradient of $f$ at $x$ for a convex function $f(x)$ while $\nabla f(x)$ denotes the gradient if it is known to be differentiable.

We use two definitions of rate of convergence for an iterative algorithm. A sequence $x^{k}$, where the superscript $k$ stands for time index, is said to converge $Q$-linearly to a point $x^{*}$ if there exists a number $v \in(0,1)$ such that $\lim _{k \rightarrow \infty} \frac{\left\|x^{k+1}-x^{*}\right\|}{\left\|x^{k}-x^{*}\right\|}=v$ with $\|\cdot\|$ being a vector norm. We say that a sequence $y^{k}$ converges $R$-linearly to $y^{*}$ if $\left\|y^{k}-y^{*}\right\| \leq\left\|x^{k}-x^{*}\right\|$ for all $k$, where $x^{k}$ converges Q-linearly to $x^{*}$.

\section{Distributed Optimization via the ADMM}

This section reviews the consensus ADMM (C-ADMM) for distributed optimization where agents can send and receive real data with infinite precision. This ideal case provides a good understanding of how the ADMM works and performs in a distributed manner. We start with the problem setting and assumptions.

\section{A. Problem Setting and Assumptions}

Throughout the paper we consider a network consisting of $N$ agents bidirectionally connected by $E$ edges, where each agent $i$ has its own objective function $f_{i}: \mathbb{R}^{M} \rightarrow \mathbb{R} \cup\{\infty\}$. Assume that the network topology is fixed. We describe this network as a symmetric directed graph $\mathcal{G}_{d}=\{\mathcal{V}, \mathcal{A}\}$ or an undirected graph $\mathcal{G}_{u}=\{\mathcal{V}, \mathcal{E}\}$, where $\mathcal{V}$ is the set of vertices with cardinality $|\mathcal{V}|=N, \mathcal{A}$ is the set of arcs with $|\mathcal{A}|=2 E$, and $\mathcal{E}$ is the set of edges with $|\mathcal{E}|=E$. Based on this graph, we would like to develop in-network algorithms that find the global optimum $\tilde{x}^{*}$ (not necessarily unique) minimizing

$$
\sum_{i=1}^{N} f_{i}(\tilde{x}) .
$$

We make the following assumptions on the local objective functions $f_{i}=g_{i}+h_{i}, i=1,2, \cdots, N$.

Assumption 1: The local objective functions are proper closed convex functions; for every $\tilde{x}$ where $f_{i}(\tilde{x})$ is well defined and $f_{i}(\tilde{x})<\infty$, there exists at least one bounded subgradient $\partial f_{i}(\tilde{x})$ such that

$$
f_{i}(\tilde{y}) \geq f_{i}(\tilde{x})+(\partial f(\tilde{x}))^{T}(\tilde{y}-\tilde{x}), \forall \tilde{y} \in \mathbb{R}^{M} .
$$

Moreover, the minimum of (1) can be attained. 
Assumption 2: The smooth components have Lipschitz continuous gradients, i.e., for each agent $i$ there exists some $M_{g_{i}}>0$ such that

$$
\left\|\nabla g_{i}(\tilde{x})-\nabla g_{i}(\tilde{y})\right\|_{2} \leq M_{g_{i}}\|\tilde{x}-\tilde{y}\|_{2}, \forall \tilde{x}, \tilde{y} \in \mathbb{R}^{M}
$$

In addition, the smooth components are strongly convex, i.e., for each agent $i$ there exists some $m_{g_{i}}>0$ such that

$$
\left(\nabla g_{i}(\tilde{x})-\nabla g_{i}(\tilde{y})\right)^{T}(\tilde{x}-\tilde{y}) \geq m_{g_{i}}\|\tilde{x}-\tilde{y}\|_{2}^{2}, \forall \tilde{x}, \tilde{y} \in \mathbb{R}^{M} .
$$

Assumption 3: The non-smooth components $h_{i}$ 's are convex.

Note that Assumption 2 implies the differentiability of $g_{i}$. Assumptions 13 together indicate that (1) has a unique and attainable solution, i.e., $\tilde{x}^{*} \in \mathbb{R}^{M}$ is unique. We only need Assumption 1 to show the convergence of the C-ADMM, while Assumption 2 is essential to establish the linear convergence when $f_{i}$ only contains the smooth component $g_{i}$.

\section{B. The ADMM for Distributed Optimization: C-ADMM}

To solve (1) using the ADMM, we first reformulate it as

$$
\begin{array}{ll}
\underset{\left\{x_{i}\right\},\left\{z_{i j}\right\}}{\operatorname{minimize}} & \sum_{i=1}^{N} f_{i}\left(x_{i}\right) \\
\text { subject to } & x_{i}=z_{i j}, x_{j}=z_{i j}, \forall(i, j) \in \mathcal{A},
\end{array}
$$

where $x_{i} \in \mathbb{R}^{M}$ is the local copy of the common optimization variable $\tilde{x}$ at agent $i$ and $z_{i j} \in \mathbb{R}^{M}$ is an auxiliary variable imposing the consensus constraint on neighboring agents $i$ and $j$. As the given network is connected, the consensus constraint ensures the consensus to be achieved over the entire network, i.e., $x_{i}=x_{j}, \forall i, j \in \mathcal{A}$, which in turn guarantees that (2) is equivalent to (1). Further define $x \in \mathbb{R}^{N M}$ as a vector concatenating all $x_{i}, z \in \mathbb{R}^{2 E M}$ as a vector concatenating all $z_{i j}, g(x)=\sum_{i=1}^{N} g_{i}\left(x_{i}\right), h(x)=\sum_{i=1}^{N} h_{i}\left(x_{i}\right)$, and $f(x)=g(x)+h(x)$. Then (2) can be written in a matrix form as

$$
\begin{aligned}
& \underset{x, z}{\operatorname{minimize}} f(x)+f^{\prime}(z) \\
& \text { subject to }
\end{aligned}
$$

where $f^{\prime}(z)=0, B=\left[-I_{2 E M} ;-I_{2 E M}\right]$ with $I_{2 E M}$ being a $2 E M \times 2 E M$ identity matrix, and $A=\left[A_{1} ; A_{2}\right]$ with $A_{1}, A_{2} \in \mathbb{R}^{2 E M \times N M}$ both being composed of $2 E \times M$ blocks of $M \times M$ matrices. If $(i, j) \in \mathcal{A}$ and $z_{i j}$ is the the $q$ th block of $z$, then the $(q, i)$ th block of $A_{1}$ and the $(q, j)$ th block of $A_{2}$ are $M \times M$ identity matrices $I_{M}$; otherwise the corresponding blocks are $M \times M$ zero matrices $0_{M}$. 
We are now ready to apply the ADMM to solving (1). The augmented Lagrangian of (3) is

$$
L_{\rho}(x, z, \lambda)=f(x)+\lambda^{T}(A x+B z)+\frac{\rho}{2}\|A x+B z\|_{2}^{2}
$$

where $\lambda=[\beta ; \gamma]$ with $\beta, \gamma \in \mathbb{R}^{2 E M}$ is the Lagrange multiplier and $\rho \in \mathbb{R}$ is a positive algorithm parameter. At iteration $k+1$, the ADMM first obtains $x^{k+1}$ by minimizing $L_{\rho}\left(x, z^{k}, \lambda^{k}\right)$, then calculates $z^{k+1}$ by minimizing $L_{\rho}\left(x^{k+1}, z, \lambda^{k}\right)$, and finally updates $\lambda^{k+1}$ from $x^{k+1}$ and $z^{k+1}$. Noting that both $A$ and $B$ are full column-rank for connected networks, Assumption 1 implies that such $x^{k+1}$ and $z^{k+1}$ exist uniquely. We then have the ADMM update given by

$$
\begin{aligned}
& x \text {-update: } \partial f\left(x^{k+1}\right)+A^{T} \lambda^{k}+\rho A^{T}\left(A x^{k+1}+B z^{k}\right)=0, \\
& z \text {-update: } B^{T} \lambda^{k}+\rho B^{T}\left(A x^{k+1}+B z^{k+1}\right)=0, \\
& \lambda \text {-update: } \lambda^{k+1}-\lambda^{k}-\rho\left(A x^{k+1}+B z^{k+1}\right)=0,
\end{aligned}
$$

where $\partial f\left(x^{k+1}\right)$ denotes a subgradient of $f(x)$ at $x=x^{k+1}$.

A nice convergence property of the ADMM, known as global convergence, states that the sequence $\left(x^{k}, z^{k}, \lambda^{k}\right)$ generated by (5) has a single limit point $\left(x^{*}, z^{*}, \lambda^{*}\right)$ under Assumption 1 Proofs can be found in [20], [21], [23]. If $\tilde{x}^{*}$ is unique (e.g., when Assumptions 13 hold), then $x^{*}=\mathbf{1}_{N} \tilde{x}^{*}$ is also unique where $\mathbf{1}_{N}=1_{N} \otimes I_{M}$, i.e., a matrix consisting of $N \times 1$ blocks of $I_{M}$. To summarize, we have

Lemma 1 (Global convergence of the ADMM [20], [21], [23]): Under Assumption 1] the updates in (5]) yield that for any initial values $x^{0} \in \mathbb{R}^{N M}, z^{0} \in \mathbb{R}^{2 E M}$ and $\lambda^{0} \in \mathbb{R}^{4 E M}$,

$$
x^{k} \rightarrow x^{*}, z^{k} \rightarrow z^{*}, \text { and } \lambda^{k} \rightarrow \lambda^{*} \text { as } k \rightarrow \infty,
$$

where $\left(x^{*}, z^{*}, \lambda^{*}\right)$ is a primal-dual solution to (4). If Assumptions 2 and 3 also hold, then $x^{*}=\mathbf{1}_{N} \tilde{x}^{*}$ is the unique solution to (3).

While (5) provides an efficient centralized algorithm to solve (3), it is not clear whether it can be carried out in a distributed manner, i.e., data exchanges only occur among neighboring nodes. Interestingly, as established in Lemma 1 the ADMM allows any initial values $x^{0}, z^{0}$ and $\lambda^{0}$ for global convergence under Assumption 1, there indeed exist initial values that decentralize (5). Define $M_{+}=A_{1}^{T}+A_{2}^{T}$ and $M_{-}=A_{1}^{T}-A_{2}^{T}$, which are respectively the extended unoriented and oriented incident matrices with respect to the directed graph $\mathcal{G}_{d} 1$ As shown in [24],

\footnotetext{
${ }^{1}$ By "extended", we mean the Kronecker product of the original matrix multiplying $I_{M}$; see [26]-[28].
} 
by initializing $\beta^{0}=-\gamma^{0}$ and $z^{0}=\frac{1}{2} M_{+}^{T} x^{0}$, the update in (5) leads to

$$
\begin{aligned}
& x_{i} \text {-update: } \partial f\left(x_{i}^{k+1}\right)+2 \rho\left|\mathcal{N}_{i}\right| x_{i}^{k+1}+\alpha_{i}^{k}-\rho\left(\left|\mathcal{N}_{i}\right| x_{i}^{k}+\sum_{j \in \mathcal{N}_{i}} x_{j}^{k}\right)=0, \\
& \alpha_{i} \text {-update: } \alpha_{i}^{k+1}=\alpha_{i}^{k}+\rho\left(\left|\mathcal{N}_{i}\right| x_{i}^{k+1}-\sum_{j \in \mathcal{N}_{i}} x_{j}^{k+1}\right)
\end{aligned}
$$

at node $i$, where $\mathcal{N}_{i}$ denotes the set of neighbors of node $i$ and $\alpha_{i}^{k} \in \mathbb{R}^{M}$ is the $i$ th block of $\alpha^{k}=M_{-} \beta^{k} \in \mathbb{R}^{N M}$.

Obviously, (6) is fully decentralized as the update of $x_{i}^{k+1}$ and $\alpha_{i}^{k+1}$ only relies on local and neighboring information.

We refer to (6) as the C-ADMM update.

\section{Linear Convergence of the C-ADMM}

Before stating the convergence results of the C-ADMM, we introduce some useful facts that are related to the undirected graph $\mathcal{G}_{u}$. These facts not only simplify our presentation but also help establish the main theorem in Section ऑII-B.

Define $L_{+}=\frac{1}{2} M_{+} M_{+}^{T}$ and $L_{-}=\frac{1}{2} M_{-} M_{-}^{T}$, which are respectively the extended signless and signed Laplacian matrices with respect to $\mathcal{G}_{u}$. Then $W=\frac{1}{2}\left(L_{+}+L_{-}\right)$is the extended degree matrix, i.e., a block diagonal matrix with its $(i, i)$ th block being the Kronecker product of $\left|\mathcal{N}_{i}\right|$ multiplying $I_{M}$ and other blocks being $0_{M}$. We have the following lemma regarding $L_{-}$.

Lemma $2([26]-[28])$ : For connected networks, $L_{-}$is positive semidefinite and always has 0 as its eigenvalue; $L_{-} b=0$ if and only if $b=\mathbf{1}_{N} \tilde{b}$ for some $\tilde{b} \in \mathbb{R}^{M}$.

As a result of the above lemma, we obtain Lemma 3 which states the one-to-one correspondence between $\alpha$ and $\beta$ provided that $\beta$ lies in the column space of $M_{-}^{T}$.

Lemma 3: Given a connected network, if $\beta$ lies in the column space of $M_{-}^{T}$, then $\alpha$ and $\beta$ are one-to-one correspondence; i.e., let $\alpha=M_{-} \beta$ and $\alpha^{\prime}=M_{-} \beta^{\prime}$ for some $\beta$ and $\beta^{\prime}$ in the column space of $M_{-}^{T}$, then $\alpha=\alpha^{\prime}$ if and only if $\beta=\beta^{\prime}$.

Proof: That $\beta=\beta^{\prime}$ implies $\alpha=\alpha^{\prime}$ is straightforward. Consider $\alpha=M_{-} \beta$ and write $\beta=M_{-}^{T} b$ for some $b \in \mathbb{R}^{N M} . \alpha^{\prime}, \beta^{\prime}$ and $b^{\prime}$ are similarly defined. Then

$$
\alpha-\alpha^{\prime}=2 L_{-}\left(b-b^{\prime}\right)=0
$$

which implies $b-b^{\prime}=\mathbf{1}_{N} \tilde{b}$ for some $\tilde{b} \in \mathbb{R}^{M}$ from Lemma 2. Since $M_{-}$is the extended oriented incident matrix with respect to $\mathcal{G}_{d}$, we have

$$
\beta-\beta^{\prime}=M_{-}^{T}\left(b-b^{\prime}\right)=\left(M_{-}^{T} \mathbf{1}_{N}\right) \tilde{b}=0 .
$$


We now turn our attention to the convergence properties of the C-ADMM. That the C-ADMM converges follows directly from global convergence of the ADMM [cf. Lemma 1]. To establish its linear convergence, we state the following lemma regarding the convergence rate of a vector concatenating $z$ and $\beta 2$

Lemma 4 ( [24 Theorem 1]): Consider the ADMM iteration (5) that solves (3). Define

$$
u=\left[\begin{array}{c}
z \\
\beta
\end{array}\right] \text { and } G=\left[\begin{array}{cc}
\rho I_{2 E M} & 0_{2 E M} \\
0_{2 E M} & \frac{1}{\rho} I_{2 E M}
\end{array}\right]
$$

with $\beta$ being a dual variable. Let $z^{0}=\frac{1}{2} M_{+}^{T} x^{0}$ and $\beta^{0}=-\gamma^{0}$, where $\gamma$ is the other dual variable and $\beta^{0}$ is initialized to lie in the column space of $M_{-}^{T}$. Suppose that Assumptions 13 hold. Then $z^{k}=\frac{1}{2} M_{+}^{T} x^{k}$ and $\beta^{k}=-\gamma^{k}$ lying in the column space of $M_{-}^{T}$ for $k=0,1, \cdots$, and $\left(x^{k}, z^{k}, \beta^{k}\right)$ converges uniquely to $\left(x^{*}, z^{*}, \beta^{*}\right)$ where $x^{*}=\mathbf{1}_{N} \tilde{x}^{*}$, $z^{*}=\frac{1}{2} M_{+}^{T} x^{*}$ and $\beta^{*}$ is a vector in the column space of $M_{-}^{T}$. If we further have $f_{i}=g_{i}$, then for any $\mu>1$, $u^{k}=\left[z^{k} ; \beta^{k}\right]$ converges Q-linearly to its optimal $u^{*}=\left[z^{*} ; \beta^{*}\right]$ with respect to the $G$-norm

$$
\left\|u^{k+1}-u^{*}\right\|_{G} \leq \frac{1}{1+\eta}\left\|u^{k}-u^{*}\right\|_{G}
$$

where $\eta=\sqrt{1+\delta}-1, m_{g} \triangleq \min _{i}\left\{m_{g_{i}}\right\}, M_{g} \triangleq \max _{i}\left\{M_{g_{i}}\right\}$, and

$$
\delta=\min \left\{\frac{(\mu-1) \tilde{\sigma}_{\min }^{2}\left(M_{-}\right)}{\mu \sigma_{\max }^{2}\left(M_{+}\right)}, \frac{4 \rho m_{g} \tilde{\sigma}_{\min }^{2}\left(M_{-}\right)}{\rho^{2} \sigma_{\max }^{2}\left(M_{+}\right) \tilde{\sigma}_{\min }^{2}\left(M_{-}\right)+\mu M_{g}^{2}}\right\} .
$$

See [24] for the proof.

Notice that when $f_{i}=g_{i}$, the non-smooth component $h_{i}=0$ and Assumption 3 is satisfied automatically. Then the above theorem indicates that $u^{k}$ is linearly convergent to the unique optimum provided that the ADMM update is initialized properly, $f_{i}$ is smooth, and Assumptions 1 and 2 hold. As an interesting observation from the ADMM iteration (5), we notice that $\left(x^{k+1}, z^{k+1}, \lambda^{k+1}\right)$ is obtained only based on $\left(z^{k}, \lambda^{k}\right)$. Given the initialization in Lemma 4. $\lambda^{k}=\left[\beta^{k} ;-\beta^{k}\right]$ for $k=0,1, \cdots$, and hence the $(k+1)$ th update only requires the knowledge of $u^{k}=\left[z^{k} ; \beta^{k}\right]$. We can then define a function $\psi(\cdot)$, which represents the update of $u^{k+1}$ from $u^{k}$ via (5), as

$$
u^{k+1}=\psi\left(u^{k}\right)
$$

Then (7) is equivalent to

$$
\left\|\psi\left(u^{k}\right)-u^{*}\right\|_{G} \leq \frac{1}{1+\eta}\left\|u^{k}-u^{*}\right\|_{G}
$$

\footnotetext{
${ }^{2}$ The linear convergence results of [22], [23] do not apply here. The step size of the dual variable update need be sufficiently small in [22] while the C-ADMM has a fixed step size $\rho$. The linear convergence result in [23] requires that $f^{\prime}(z)$ is strongly convex or $B$ is full row-rank. However, in our formulation [3], $f^{\prime}(z)=0$ is not strongly convex and $B=\left[-I_{2 E M} ;-I_{2 E M}\right]$ is row-rank deficient.
} 
Compared with the initialization conditions that lead the ADMM to the C-ADMM, we notice an extra initialization condition that $\beta_{0}$ lies in the column space of $M_{-}^{T}$ in Lemma 4 Without this condition, $x^{k}$ still converges to its unique optimal value $x^{*}=1_{N} \tilde{x}^{*}$ under Assumptions 1 13, whereas the uniqueness of $\beta^{*}$ may not hold nor the linear convergence of $u^{k}$. While this condition implies that $\alpha^{0}$ in the C-ADMM is initialized in the column space of $L_{-}$, it is not clear whether such $\alpha^{0}$ ensures the linear convergence of the C-ADMM. In the following we show that the C-ADMM indeed converges linearly to its optimal value under Assumptions 1 and 2 given that $f_{i}=g_{i}$ and $\alpha^{0}$ lies in the column space of $L_{-}$. To see this, let $x^{0}$ and $\alpha^{0}$ be the initial values in the C-ADMM where $\alpha^{0}$ is in the column space of $L_{-}$. Lemma 3 implies that there exists a unique $\beta^{0}$ in the column space of $M_{-}^{T}$ such that $\alpha^{0}=M_{-} \beta^{0}$. This $\beta^{0}$ together with $z^{0}=\frac{1}{2} M_{+}^{T} x^{0}$ and $\gamma^{0}=-\beta^{0}$ leads the ADMM to the C-ADMM with exactly the same initial values $x^{0}$ and $\alpha^{0}$. Then $x^{k}$ stays the same in the ADMM and the C-ADMM iterations, and $\alpha^{k}=M_{-} \beta^{k}$ where $\beta^{k}$ is the $k$-th update in the ADMM. As such, we can study the convergence rate of the C-ADMM through the ADMM that is correspondingly initialized. The linear convergence of the C-ADMM is presented below.

Theorem 1 (Linear convergence of the $C$-ADMM): Let $f_{i}=g_{i}$ and consider the C-ADMM update (6). Suppose that Assumptions 1 and 2 hold. If we initialize $\alpha^{0}$ in the column space of $L_{-}$, then $\left[x^{k} ; \alpha^{k}\right]$ converges R-linearly to its optimal value $\left[x^{*} ; \alpha^{*}\right]$ where $x^{*}=\mathbf{1}_{N} \tilde{x}^{*}$ and $\alpha^{*}=\nabla g\left(\mathbf{1}_{N} \tilde{x}^{*}\right)$.

Proof: From Lemma 3, we can denote by $\beta^{0}$ the unique vector in the column space of $M_{-}^{T}$ such that $\alpha^{0}=M_{-} \beta_{0}$. Consider the ADMM iteration (5) with initialization $z^{0}=\frac{1}{2} M_{+}^{T} x^{0}$ and $\lambda^{0}=\left[\beta^{0} ;-\beta^{0}\right]$. Then the convergence of $x^{k}$ to $x^{*}=\mathbf{1}_{N} \tilde{x}^{*}$ follows from Lemma 1 By plugging $x_{i}^{*}=\tilde{x}^{*}$ into (6), we obtain $\alpha_{i}^{*}=\nabla g_{i}\left(\tilde{x}^{*}\right)$.

To show the linear convergence, we first have from [24, Equation (29)] that

$$
\left\|x^{k+1}-x^{*}\right\|_{2}^{2} \leq \frac{1}{m_{g}}\left\|u^{k}-u^{*}\right\|_{G}^{2}
$$

where $m_{g}$ and $u^{k}$ are defined in Lemma 4, Recalling the definition of $G$, we have

$$
\begin{aligned}
\left\|\alpha^{k+1}-\alpha^{*}\right\|_{2}^{2} & =\left\|M_{-}\left(\beta^{k+1}-\beta^{*}\right)\right\|_{2}^{2} \\
& \leq \sigma_{\max }^{2}\left(M_{-}\right) \rho\left\|u^{k+1}-u^{*}\right\|_{G}^{2} \\
& \stackrel{(a)}{\leq} \sigma_{\max }^{2}\left(M_{-}\right) \frac{\rho}{(1+\eta)^{2}}\left\|u^{k}-u^{*}\right\|_{G}^{2},
\end{aligned}
$$

where $(a)$ is from the linear convergence of $u^{k}$ as the initialization conditions in Lemma 4 are satisfied. Therefore,

$$
\left\|\left[\begin{array}{c}
x^{k+1} \\
\alpha^{k+1}
\end{array}\right]-\left[\begin{array}{c}
x^{*} \\
\alpha^{*}
\end{array}\right]\right\|_{2}^{2} \leq\left(\frac{1}{m_{g}}+\sigma_{\max }^{2}\left(M_{-}\right) \frac{\rho}{(1+\eta)^{2}}\right)\left\|u^{k}-u^{*}\right\|_{G}^{2},
$$

which establishes the R-linear convergence of $\left[x^{k} ; \alpha^{k}\right]$ since $u^{k}$ converges Q-linearly to $u^{*}$. 


\section{QuAnTIZED CONSEnsus ADMM}

To model the effect of quantized communication, we assume that each agent can store and compute real values with infinite precision; an agent, however, can only transmit quantized data through the channel which are received by its neighbors without any error. Given a quantization resolution $\Delta>0$, define the quantization lattice in $\mathbb{R}$ by

$$
\Lambda=\{t \Delta: t \in \mathbb{Z}\}
$$

A quantizer is a function $Q: \mathbb{R} \rightarrow \Lambda$ that maps a real value to some point in $\Lambda$. Among all deterministic quantizers, we consider the rounding quantizer that projects $y \in \mathbb{R}$ to its nearest point in $\Lambda$ :

$$
Q(y)=t \Delta, \text { if }\left(t-\frac{1}{2}\right) \Delta \leq y<\left(t+\frac{1}{2}\right) \Delta .
$$

By quantizing a vector we mean quantizing each of its entries. For $w \in \mathbb{R}^{L}, L \in \mathbb{Z}^{+}$, the rounding quantizer projects $w$ to its nearest point in $\Lambda^{L}$, and we use $w_{[Q]}$ to denote the quantizer output of $w$. Define $e=w_{[Q]}-w$ as the quantization error. It is clear that for any $w \in \mathbb{R}^{L}$,

$$
\|e\|_{2} \leq \frac{1}{2} \Delta \sqrt{L}
$$

We next use the above rounding quantization to modify the C-ADMM to meet the communication constraint, resulting in the quantized consensus ADMM (QC-ADMM) in Algorithm 1

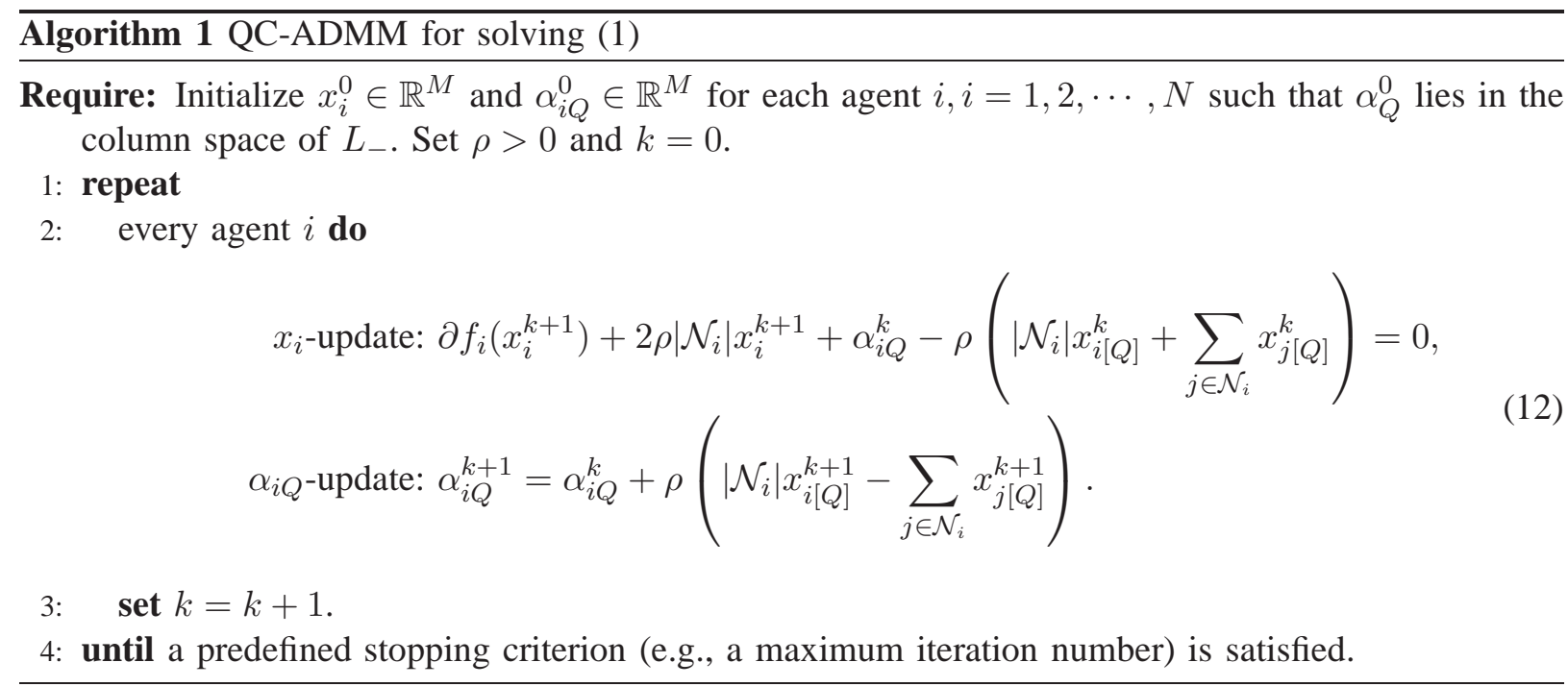

In Algorithm 1 we use the subscript $Q$ to differentiate between the QC-ADMM and C-ADMM updates, and $\alpha_{i Q}^{k}$ is not necessarily equal to $\alpha_{i[Q]}^{k}$. Note that $x_{i}^{k}$ is quantized at its own node for the $(k+1)$ th update; the reason will be given in Remark 4 We will establish the connection of the QC-ADMM with the standard ADMM in Section ЩI-A and the convergence results of the QC-ADMM in Sections ЩI-B and 


\section{A. Connection with the ADMM}

Now the QC-ADMM update (12) seems to be a direct modification from the C-ADMM update by quantizing $x^{k}$ for the $(k+1)$ th update; it is not clear how it relates to the standard ADMM. We will show that (12) can be derived from (5) by imposing a quantization operation on $x$ immediately after the $x$-update, i.e.,

$$
\begin{aligned}
x \text {-update }: & \partial f\left(x^{k+1}\right)+A^{T} \lambda_{Q}^{k}+\rho A^{T}\left(A x^{k+1}+B z_{Q}^{k}\right)=0, \\
x_{[Q]} \text {-update }: & x_{[Q]}^{k+1}=Q\left(x^{k+1}\right), \\
z_{Q} \text {-update }: & B^{T} \lambda_{Q}^{k}+\rho B^{T}\left(A x_{[Q]}^{k+1}+B z_{Q}^{k+1}\right)=0, \\
\lambda_{Q} \text {-update }: & \lambda_{Q}^{k+1}-\lambda_{Q}^{k}-\rho\left(A x_{[Q]}^{k+1}+B z_{Q}^{k+1}\right)=0,
\end{aligned}
$$

where the subscript $Q$ is adopted to differentiate between the updates before and after the $\left.x_{[Q]}\right]^{\text {-update. Again we }}$ do not have $z_{Q}^{k}=z_{[Q]}^{k}$ or $\lambda_{Q}^{k}=\lambda_{[Q]}^{k}$ in general. Since $\left(x^{k+1}, z_{Q}^{k+1}, \lambda_{Q}^{k+1}\right)$ is updated only based on $\left(z_{Q}^{k}, \lambda_{Q}^{k}\right)$ given the deterministic quantization operation defined by (10), we can still perform the $\lambda$-update and $z$-update before the

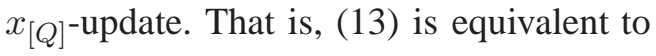

$$
\begin{array}{cl}
x \text {-update }: & \partial f\left(x^{k+1}\right)+A^{T} \lambda_{Q}^{k}+\rho A^{T}\left(A x^{k+1}+B z_{Q}^{k}\right)=0, \\
z \text {-update }: & B^{T} \lambda_{Q}^{k}+\rho B^{T}\left(A x^{k+1}+B z^{k+1}\right)=0, \\
\lambda \text {-update }: & \lambda^{k+1}-\lambda_{Q}^{k}-\rho\left(A x^{k+1}+B z^{k+1}\right)=0, \\
x_{[Q]} \text {-update }: & x_{[Q]}^{k+1}=Q\left(x^{k+1}\right), \\
z_{Q} \text {-update }: & B^{T} \lambda_{Q}^{k}+\rho B^{T}\left(A x_{[Q]}^{k+1}+B z_{Q}^{k+1}\right)=0, \\
\lambda_{Q} \text {-update }: & \lambda_{Q}^{k+1}-\lambda_{Q}^{k}-\rho\left(A x_{[Q]}^{k+1}+B z_{Q}^{k+1}\right)=0 .
\end{array}
$$

With this formulation, we can use similar approaches in [10], [24] to show that (13) leads to (12) if $\lambda_{Q}^{0}$ and $z_{Q}^{0}$ are properly initialized. First multiplying the two sides of (14f) by $B^{T}$ and adding it to (14e), we have

$$
\begin{aligned}
B^{T} \lambda_{Q}^{k+1} & =0 \\
B^{T} \lambda_{Q}^{k}+\rho B^{T}\left(A x_{[Q]}^{k+1}+B z_{Q}^{k+1}\right) & =0 .
\end{aligned}
$$

Recalling $\lambda_{Q}=\left[\beta_{Q} ; \gamma_{Q}\right]$ and $B=\left[-I_{2 E M} ;-I_{2 E M}\right]$, if we initialize $\beta_{Q}^{0}=-\gamma_{Q}^{0}$, then $\beta_{Q}^{k}=-\gamma_{Q}^{k}$ for $k=0,1, \cdots$, and (15b) implies $z_{Q}^{k+1}=\frac{1}{2} M_{+}^{T} x_{[Q]}^{k+1}$. By initializing $z_{Q}^{0}=\frac{1}{2} M_{+}^{T} x_{[Q]}^{0}$, we have $z_{Q}^{k}=\frac{1}{2} M_{+}^{T} x_{[Q]}^{k}$ for $k=0,1, \cdots$. To summarize, with the initialization $\beta_{Q}^{0}=-\gamma_{Q}^{0}$ and $z_{Q}^{0}=\frac{1}{2} M_{+}^{T} x_{[Q]}^{0}$, 15a and (15b) are equivalent to

$$
\begin{aligned}
B^{T} \lambda_{Q}^{k} & =0, \\
z_{Q}^{k}-\frac{1}{2} M_{+}^{T} x_{[Q]}^{k} & =0 .
\end{aligned}
$$


Next we consider updates 114a-14c). Multiplying the two sides of the $\lambda$-update by $A^{T}$ and $B^{T}$ and adding them to the $x$-update and $z$-update respectively, we get $\partial f\left(x^{k+1}\right)+A^{T} \lambda^{k+1}+\rho A^{T} B\left(z_{Q}^{k}-z^{k+1}\right)=0$ and $B^{T} \lambda^{k+1}=0$. Therefore, (14a)-(14c) can be equivalently expressed as

$$
\begin{aligned}
\partial f\left(x^{k+1}\right)+A^{T} \lambda^{k+1}+\rho A^{T} B\left(z_{Q}^{k}-z^{k+1}\right) & =0, \\
B^{T} \lambda^{k+1} & =0, \\
\lambda^{k+1}-\lambda_{Q}^{k}-\rho\left(A x^{k+1}+B z^{k+1}\right) & =0 .
\end{aligned}
$$

Also by the definitions of $\lambda$ and $B$, we know that $\beta^{k+1}=\gamma^{k+1}$ from (17b), and that 17a splits into two equations $\beta^{k+1}-\beta_{Q}^{k}-\rho A_{1} x^{k+1}+\rho z^{k+1}=0$ and $\gamma^{k+1}-\gamma_{Q}^{k}-\rho A_{2} x^{k+1}+\rho z^{k+1}=0$. Summing and subtracting these two equations lead to $z^{k+1}-\frac{1}{2} M_{+}^{T} x^{k+1}=0$ and $\beta^{k+1}-\beta_{Q}^{k}-\frac{\rho}{2} M_{-}^{T} x^{k+1}=0$, respectively. Thus, (17a)-(17c) reduce to

$$
\begin{aligned}
\partial f\left(x^{k+1}\right)+A^{T} \lambda^{k+1}+\rho A^{T} B\left(z_{Q}^{k}-z^{k+1}\right) & =0, \\
z^{k+1}-\frac{1}{2} M_{+}^{T} x^{k+1} & =0, \\
\beta^{k+1}-\beta_{Q}^{k}-\frac{\rho}{2} M_{-}^{T} x^{k+1} & =0 .
\end{aligned}
$$

By substituting (16b) and (18b) into (18a), we have (14a)-(14f) finally equivalent to

$$
\begin{aligned}
\partial f\left(x^{k+1}\right)+M_{-} \beta^{k+1}-\frac{\rho}{2} M_{+} M_{+}^{T} x_{[Q]}^{k}+\frac{\rho}{2} M_{+} M_{+}^{T} x^{k+1} & =0, \\
\beta^{k+1}-\beta_{Q}^{k}-\frac{\rho}{2} M_{-}^{T} x^{k+1} & =0, \\
\beta_{Q}^{k+1}-\beta_{Q}^{k}-\frac{\rho}{2} M_{-}^{T} x_{[Q]}^{k+1} & =0 .
\end{aligned}
$$

If we further multiply the two sides of $19 \mathrm{~b}$ by $-M_{-}$and add it to $19 \mathrm{a}$, we obtain

$$
\partial f\left(x^{k+1}\right)+M_{-} \beta_{Q}^{k}+\frac{\rho}{2}\left(M_{+} M_{+}^{T}+M_{-} M_{-}^{T}\right) x^{k+1}-\frac{\rho}{2} M_{+} M_{+}^{T} x_{[Q]}^{k}=0 .
$$

From (20), we see that the update of $x^{k+1}$ relies on $M_{-} \beta_{Q}^{k}$ instead of $\beta_{Q}^{k}$. Hence, multiplying both sides of (19c) by $M_{-}$yields

$$
M_{-} \beta_{Q}^{k+1}-M_{-} \beta_{Q}^{k}-\frac{\rho}{2} M_{-} M_{-}^{T} x_{[Q]}^{k+1}=0
$$

Letting $\alpha_{Q}^{k}=M_{-} \beta_{Q}^{k}$ and recalling the definitions of $L_{-}, L_{+}$and $W$, we have (20) and (21) equivalent to

$$
\begin{aligned}
x \text {-update }: & \partial f\left(x^{k+1}\right)+2 \rho W x^{k+1}+\alpha_{Q}^{k}-\rho L_{+} x_{[Q]}^{k}=0, \\
\alpha_{Q} \text {-update }: & \alpha_{Q}^{k+1}-\alpha_{Q}^{k}-\rho L_{-} x_{[Q]}^{k+1}=0,
\end{aligned}
$$


which is exactly the matrix form of (12).

\section{B. Convergence Results: Smooth Objective Functions}

For ease of presentation, we first study a simple case where objective functions only contain the smooth components, i.e., $f_{i}=g_{i}$. Then $\partial f_{i}=\nabla g_{i}$ under Assumptions 1 and 2

We consider the effect of the rounding quantization by writing

$$
x_{[Q]}^{k+1}=x^{k+1}+e^{k+1} .
$$

Then the $\alpha_{Q}$-update in the QC-ADMM iteration (22) is equivalent to

$$
\alpha_{Q}^{k+1}=\alpha_{Q}^{k}+\rho L_{-} x^{k+1}+\rho L_{-} e^{k+1}
$$

Compared with the C-ADMM, we see that $\left[x_{[Q]}^{k+1} ; \alpha_{Q}^{k+1}\right]$ is obtained by performing the C-ADMM update on $\left[x_{[Q]}^{k} ; \alpha_{Q}^{k}\right]$ followed by adding an error term $\left[e^{k+1} ; \rho L_{-} e^{k+1}\right]$ which is caused by the quantization operation. Due to the possible nonlinearity of $\nabla g_{i}$, we cannot easily write $\left[x_{[Q]}^{k+1} ; \alpha_{Q}^{k+1}\right]$ as the sum of the $(k+1)$ th C-ADMM update plus an accumulated error term. Instead, we utilize the vector $u_{Q}^{k}=\left[z_{Q}^{k} ; \beta_{Q}^{k}\right]$ to study the convergence of the QC-ADMM.

From Section [II-A we know that the QC-ADMM can be obtained from (14a)- 14f by initializing $\beta_{Q}^{0}=-\gamma_{Q}^{0}$ and $z_{Q}^{0}=\frac{1}{2} M_{+}^{T} x_{[Q]}^{0}$. This initialization then leads to $\beta_{Q}^{k}=-\gamma_{Q}^{k}, z_{Q}^{k}=\frac{1}{2} M_{+}^{T} x_{[Q]}^{k}, \beta^{k+1}=-\gamma^{k+1}$, and $z^{k+1}=\frac{1}{2} M_{+}^{T} x^{k}$ for $k=0,1, \cdots$. Therefore, the $(k+1)$ th update using (14a)-(14f) is only based on $\left[z_{Q}^{k} ; \beta_{Q}^{k}\right]$. Similar to studying the C-ADMM through the correspondingly initialized ADMM, we investigate the updats of $\left[z_{Q}^{k+1} ; \beta_{Q}^{k+1}\right]$ through (14a)-114f) that are also correspondingly initialized. To this end, we notice the following relation between $z_{Q}^{k+1}$ and $z^{k+1}$ :

$$
z_{Q}^{k+1}=\frac{1}{2} M_{+}^{T} x_{[Q]}^{k+1}=\frac{1}{2} M_{+}^{T} x^{k+1}+\frac{1}{2} M_{+}^{T} e^{k+1}=z^{k+1}+\frac{1}{2} M_{+}^{T} e^{k+1} .
$$

Combining (19b) and (19c), we also obtain

$$
\beta_{Q}^{k+1}=\beta^{k+1}+\frac{\rho}{2} M_{-}^{T} e^{k+1}
$$

Since $\left[z^{k+1} ; \beta^{k+1}\right]$ is obtained by performing a standard ADMM update on $\left[z_{Q}^{k} ; \beta_{Q}^{k}\right]$ as seen from (14a)-(14c), we can represent the update of $u_{Q}^{k+1}$ from $u_{Q}^{k}$ as

$$
u_{Q}^{k+1}=\psi\left(u_{Q}^{k}\right)+u_{e}^{k}
$$

where $u_{e}^{k}=\left[\frac{1}{2} M_{+}^{T} e^{k+1} ; \frac{\rho}{2} M_{-}^{T} e^{k+1}\right]$, and $\psi$ denotes the standard ADMM update as defined by (8). We will use this relation to write $u_{Q}^{k+1}$ as the sum of the $(k+1)$ th $\mathrm{ADMM}$ update from $u_{Q}^{0}$ plus an accumulated error term 
caused by quantization. If the QC-ADMM starts with $\alpha_{Q}^{0}$ which is in the column space $L_{-}$, then the $\alpha_{Q^{-}}$update implies that $\alpha_{Q}^{k}$ lies in the column space of $L_{-}$for $k=0,1, \cdots$. Therefore, the corresponding ADMM update possesses the linear convergence rate [cf. Equation [9] as discussed in Section [I-C]. Utilizing this property we are able to establish the absolute convergence and hence the convergence of the accumulated error term. We first state the boundedness of $u_{e}^{k}$ and $u_{Q}^{k}$ in the following lemma.

Lemma 5: Let $f_{i}=g_{i}$ and suppose that Assumptions 1 and 2 hold. Consider the QC-ADMM algorithm. Let $\beta_{Q}^{0}$ be the unique vector in the column space of $M_{-}$such that $\alpha_{Q}^{0}=M_{-}^{T} \beta_{Q}^{0}$. Let also $z_{Q}^{0}=\frac{1}{2} M_{+}^{T} x_{[Q]}^{0}$ and $\gamma_{Q}^{0}=-\beta_{Q}^{0}$ in (14a)-114f). Then for $k=0,1, \cdots$,

$$
\left\|u_{e}^{k}\right\|_{G} \leq \tau_{0} \text { and }\left\|u_{Q}^{k}-u^{*}\right\|_{G} \leq\left\|u_{Q}^{0}-u^{*}\right\|_{G}+\left(1+\frac{1}{\eta}\right) \tau_{0}
$$

where $u^{*}=\left[\mathbf{1}_{2 E} \tilde{x}^{*} ; \beta^{*}\right]$ with $\mathbf{1}_{2 E}=1_{2 E} \otimes I_{M}$ and $\beta^{*}$ being the unique vector in the column space of $M_{-}$such that $M_{-}^{T} \beta^{*}=\alpha^{*}=\nabla g\left(\mathbf{1}_{N} \tilde{x}^{*}\right), \tau_{0}=\frac{1}{4} \Delta \sqrt{\rho M\left(\sigma_{\max }^{2}\left(M_{+}\right)+\sigma_{\max }^{2}\left(M_{-}\right)\right)}$, and $\eta$ is defined in Lemma 4

Proof: The boundedness of $u_{e}^{k}$ follows directly from the boundedness of $e^{k+1}$, i.e.,

$$
\begin{aligned}
\left\|u_{e}^{k}\right\|_{G}^{2} & =\rho\left\|\frac{1}{2} M_{+}^{T} e^{k+1}\right\|_{2}^{2}+\frac{1}{\rho}\left\|\frac{1}{2} \rho M_{-}^{T} e^{k+1}\right\|_{2}^{2} \\
& \leq \frac{1}{4} \rho\left(\sigma_{\max }^{2}\left(M_{+}\right)+\sigma_{\max }^{2}\left(M_{-}\right)\right)\left\|e^{k+1}\right\|_{2}^{2} \\
& \leq \frac{1}{16} \rho M \Delta^{2}\left(\sigma_{\max }^{2}\left(M_{+}\right)+\sigma_{\max }^{2}\left(M_{-}\right)\right) . \\
& =\tau_{0}^{2} .
\end{aligned}
$$

Since $\alpha_{Q}^{0}$ is initialized in the column space of $L_{-}$, we see that $\alpha_{Q}^{k}$ also lies in the column space of $L_{-}$from the $\alpha_{Q}$-update of the QC-ADMM. Then using (7) and (9), we have

$$
\begin{aligned}
\left\|u_{Q}^{k+1}-u^{*}\right\|_{G}= & \left\|\psi\left(u_{Q}^{k}\right)+u_{e}^{k}-u^{*}\right\|_{G} \\
\leq & \left\|\psi\left(u_{Q}^{k}\right)-u^{*}\right\|_{G}+\left\|u_{e}^{k}\right\|_{G} \\
\leq & \frac{1}{1+\eta}\left\|u_{Q}^{k}-u^{*}\right\|_{G}+\left\|u_{e}^{k}\right\|_{G} \\
& \cdots \\
\leq & \frac{1}{(1+\eta)^{k+1}}\left\|u_{Q}^{0}-u^{*}\right\|_{G}+\sum_{j=0}^{k} \frac{1}{(1+\eta)^{j}}\left\|u_{e}^{j}\right\|_{G} .
\end{aligned}
$$

Therefore, for $k=0,1, \cdots$, we have

$$
\left\|u_{Q}^{k}-u^{*}\right\|_{G} \leq\left\|u_{Q}^{0}-u^{*}\right\|_{G}+\sum_{i=0}^{\infty} \frac{1}{(1+\eta)^{i}} \tau_{0}=\left\|u_{Q}^{0}-u^{*}\right\|_{G}+\left(1+\frac{1}{\eta}\right) \tau_{0} .
$$


With this lemma, we are ready to establish our main theorem as follows.

Theorem 2: Let $f_{i}=g_{i}$. Consider the QC-ADMM iteration (12), and suppose that Assumptions 11 and 2 hold. Initializing $\alpha_{Q}^{0}$ in the column space of $L_{-}$, we have

1) Convergence: the sequence $\left(x_{[Q]}^{k}, \alpha_{Q}^{k}\right)$ generated by (12) converges to a finite value $\left(\mathbf{1}_{N} \tilde{x}_{Q}^{*}, \alpha_{Q}^{*}\right)$ as $k \rightarrow \infty$, where $\tilde{x}_{Q}^{*}$ is some vector in $\Lambda^{M}$ and not necessarily equal to $\tilde{x}_{[Q]}^{*}$.

2) Consensus error: an upper bound for the consensus error is given by

$$
\left\|\tilde{x}_{Q}^{*}-\tilde{x}^{*}\right\|_{2} \leq\left(\frac{1}{2}+\rho \frac{2 E}{\sum_{i=1}^{N} m_{g_{i}}}\right) \sqrt{M} \Delta .
$$

3) Number of iterations: $\left(x_{[Q]}^{k}, \alpha_{Q}^{k}\right)$ converges within $\left\lceil\log _{1+\eta} \Omega\right\rceil$ iterations, where

$$
\Omega=\max \left\{\frac{3 \sqrt{\rho} \sigma_{\max }\left(M_{-}\right)(1+\eta)^{2}\left(\left\|u_{Q}^{0}-u^{*}\right\|_{G}+\tau_{0}\right)}{\eta \Delta}, \frac{3(1+\eta)\left(\left\|u_{Q}^{0}-u^{*}\right\|_{G}+\tau_{0}\right)}{\sqrt{2 \rho E} \eta \Delta}\right\},
$$

and $\lceil y\rceil, y \in \mathbb{R}$, means the smallest integer that is greater than or equal to $y$.,

Proof: We prove the three claims one by one.

Convergence: From (14a)-114f), we know that $x^{k+1}$ is updated only based on $u_{Q}^{k}=\left[z_{Q}^{k} ; \beta_{Q}^{k}\right]$ if the updates are initialized with $\beta_{Q}^{0}=-\gamma_{Q}^{0}$ and $z_{Q}^{0}=\frac{1}{2} M_{-}^{T} x_{[Q]}^{0}$. As long as $u_{Q}^{k}$ converges, we must have $x_{[Q]}^{k+1}$ and $\alpha_{Q}^{k+1}$ converging. Given that $\alpha_{Q}^{k}$ converges, $L_{-} x_{Q}^{k}$ must converge to 0 due to the $\alpha_{Q}$-update, and hence $x_{[Q]}^{k}$ converges to a consensus by Lemma 2 Therefore, to prove the convergence of $\left(x_{[Q]}^{k}, \alpha_{Q}^{k}\right)$, it is enough to show the convergence of $u_{Q}^{k}$.

Following (23), we have

$$
\begin{aligned}
u_{Q}^{k+1} & =\psi\left(u_{Q}^{k}\right)+u_{e}^{k} \\
& =\psi\left(\psi\left(u_{Q}^{k-1}\right)+u_{e}^{k-1}\right)+u_{e^{\prime}}^{k} \\
& =\psi^{2}\left(u_{Q}^{k-1}\right)+u_{e^{\prime}}^{k-1}+u_{e^{\prime}}^{k} \\
& =\cdots \\
& =\psi^{k+1}\left(u_{Q}^{0}\right)+\sum_{i=0}^{k} u_{e^{\prime}}^{k-i},
\end{aligned}
$$

where $\psi^{i}(\cdot), i=0,1, \cdots$, denotes the $i$-th standard ADMM update on its argument and $u_{e^{\prime}}^{k-i}=\psi^{i}\left(\psi\left(u^{k-i}\right)+u_{e}^{k-i}\right)-$ $\psi^{i+1}\left(u^{k-i}\right)$. We only need to prove the convergence of the accumulated error term $\sum_{i=0}^{k} u_{e^{\prime}}^{k-i}$ as the first term is the $(k+1)$ th standard ADMM update which converges to $u^{*}$ as $k \rightarrow \infty$. It then suffices to show the boundedness of $\lim _{k \rightarrow \infty} \sum_{i=0}^{k}\left\|u_{e^{\prime}}^{k-i}\right\|_{G}$ due to the comparison theorem and the fact that absolute convergence implies convergence 
[29]. We first obtain an upper bound on $\left\|u_{e^{\prime}}^{k-i}\right\|_{G}$ :

$$
\begin{aligned}
\left\|u_{e^{\prime}}^{k-i}\right\|_{G} & =\left\|\psi^{i}\left(\psi\left(u_{Q}^{k-i}\right)+u_{e}^{k-i}\right)-\psi^{i+1}\left(u_{Q}^{k-i}\right)\right\|_{G} \\
& =\left\|\psi^{i}\left(\psi\left(u_{Q}^{k-i}\right)+u_{e}^{k-i}\right)-u^{*}-\left(\psi^{i+1}\left(u_{Q}^{k-i}\right)-u^{*}\right)\right\|_{G} \\
& \leq\left\|\psi^{i}\left(\psi\left(u_{Q}^{k-i}\right)+u_{e}^{k-i}\right)-u^{*}\right\|_{G}+\left\|\psi^{i+1}\left(u_{Q}^{k-i}\right)-u^{*}\right\|_{G} \\
& \stackrel{(a)}{\leq} \frac{1}{(1+\eta)^{i}}\left\|\psi\left(u_{Q}^{k-i}\right)+u_{e}^{k-i}-u^{*}\right\|_{G}+\frac{1}{(1+\eta)^{i+1}}\left\|u_{Q}^{k-i}-u^{*}\right\|_{G} \\
& \leq \frac{1}{(1+\eta)^{i}}\left\|\psi\left(u_{Q}^{k-i}\right)-u^{*}\right\|_{G}+\frac{1}{(1+\eta)^{i}}\left\|u_{e}^{k-i}\right\|_{G}+\frac{1}{(1+\eta)^{i+1}}\left\|u_{Q}^{k-i}-u^{*}\right\|_{G} \\
& \leq \frac{2}{(1+)}{ }^{(1+\eta)^{i+1}}\left\|u_{Q}^{0}-u^{*}\right\|_{G}+\frac{3}{(1+\eta)^{i}},
\end{aligned}
$$

where $(a)$ is from Lemma 4 and $(b)$ is due to Lemma 5 Therefore,

$$
\lim _{k \rightarrow \infty} \sum_{i=0}^{k}\left\|u_{e^{\prime}}^{k-i}\right\|_{G} \leq \sum_{i=0}^{\infty} \frac{2}{(1+\eta)^{i+1}}\left\|u_{Q}^{0}-u^{*}\right\|_{G}+\sum_{i=0}^{\infty} \frac{3}{(1+\eta)^{i}} \tau_{0} .
$$

The convergence proof is complete by noting that $\eta>0$.

Consensus error: The consensus error may be studied directly by calculating the accumulated error term in (27). However, the bound in (29) is quite loose in general as the bounds in Lemmas 4 and 5 are themselves loose for the respective quantities. We alternatively study the QC-ADMM iteration (12) using the fact that $x_{[Q]}^{k}$ converges to a consensus as $k \rightarrow \infty$.

Let $\tilde{x}_{Q}^{*} \in \Lambda^{M}$ be the convergent quantized value at each agent. Then $x_{i[Q]}^{\infty}=\tilde{x}_{Q}^{*}$ and $x_{i}^{\infty}=\tilde{x}_{Q}^{*}-e_{i}^{*}$ for $i=1,2, \cdots, N$, where $e_{i}^{*}$ is the quantization error at agent $i$. It is important to note that $\tilde{x}_{Q}^{*}$ does not represent the quantized value of the global optimum $\tilde{x}^{*}$, i.e., $\tilde{x}_{Q}^{*}$ is not necessarily equal to $\tilde{x}_{[Q]}^{*}$. Summing up both sides of (12) from $i=1$ to $N$, we have

$$
\sum_{i=1}^{N}\left(\nabla g_{i}\left(\tilde{x}_{Q}^{*}-e_{i}^{*}\right)+2 \rho\left|\mathcal{N}_{i}\right|\left(\tilde{x}_{Q}^{*}-e_{i}^{*}\right)\right)=\sum_{i=1}^{N}\left(\left|\mathcal{N}_{i}\right| \tilde{x}_{Q}^{*}+\rho \sum_{j \in \mathcal{N}_{i}} \tilde{x}_{Q}^{*}\right) .
$$

Here we use the fact that $\alpha_{Q}^{k}$ lies in the column space of $L_{-}$, i.e., $\alpha_{Q}^{k}=L_{-} b^{k}$ for some $b^{k} \in \mathbb{R}^{N M}$. By Lemma 2. we have

$$
\sum_{i=1}^{N} \alpha_{i Q}^{k}=\left(L_{-} b^{k}\right)^{T} \mathbf{1}_{N}=\left(b^{k}\right)^{T}\left(L_{-}^{T} \mathbf{1}_{N}\right)=0 .
$$

Since $f_{i}=g_{i}$ which is differentiable and strongly convex in $\mathbb{R}^{M}, \tilde{x}^{*}$ is the solution to problem (1) if and only if

$$
\sum_{i=1}^{N} \nabla g_{i}\left(\tilde{x}^{*}\right)=0
$$


Thus, (30) leads to

$$
\sum_{i=1}^{N}\left(\nabla g_{i}\left(\tilde{x}_{Q}^{*}-e_{i}^{*}\right)-\nabla g_{i}\left(\tilde{x}^{*}\right)\right)=\sum_{i=1}^{N} 2 \rho\left|\mathcal{N}_{i}\right| e_{i}^{*}
$$

Recalling the strong convexity assumption, we have

$$
\left\|\nabla g_{i}\left(\tilde{x}_{Q}^{*}-e_{i}^{*}\right)-\nabla g_{i}\left(\tilde{x}^{*}\right)\right\|_{2} \geq m_{g_{i}}\left\|\tilde{x}_{Q}^{*}-e_{i}^{*}-\tilde{x}^{*}\right\|_{2} .
$$

Together with (11), we obtain the following upper bound

$$
\left\|\tilde{x}_{Q}^{*}-\tilde{x}^{*}\right\|_{2} \leq\left(\frac{1}{2}+\rho \frac{2 E}{\sum_{i=1}^{N} m_{g_{i}}}\right) \sqrt{M} \Delta .
$$

We next use an example to show that this bound is indeed tight. Consider a simple two-node network where $f_{1}(\tilde{x})=\left(\tilde{x}+\frac{3}{2}\right)^{2}$ and $f_{2}(\tilde{x})=\left(\tilde{x}+\frac{7}{2}\right)^{2}, \tilde{x} \in \mathbb{R}$. Then $m_{f_{1}}=m_{f_{2}}=1$ and $\tilde{x}^{*}=-\frac{5}{2}$. Set both $\Delta$ and $\rho$ to be 1 . In this case, we have $M=1, N=2, E=1$ and

$$
L_{-}=\left[\begin{array}{cc}
1 & -1 \\
-1 & 1
\end{array}\right]
$$

We start with $x_{1[Q]}^{0}=x_{2[Q]}^{0}=-1$ and $\alpha_{1 Q}^{0}=-\alpha_{2 Q}^{0}=1$. One can easily check that $\alpha_{Q}^{0}=\left[\alpha_{1 Q}^{0} ; \alpha_{2 Q}^{0}\right]$ lies in the column space of $L_{-}$, and that $x_{1[Q]}^{k}=x_{2[Q]}^{k}=-1$ and $\alpha_{1 Q}^{k}=-\alpha_{2 Q}^{k}=1$ for $k=0,1, \cdots$, in the updates of (12). Hence the consensus error is

$$
\left\|\tilde{x}_{Q}^{*}-\tilde{x}^{*}\right\|_{2}=\frac{3}{2}=\left(\frac{1}{2}+\rho \frac{2 E}{\sum_{i=1}^{N} m_{f_{i}}}\right) \sqrt{M} \Delta,
$$

Number of iterations: The convergence of $\left(x_{[Q]}^{k}, \alpha_{Q}^{k}\right)$ implies that there exists a finite $k_{0}$ such that $\left\|\alpha_{Q}^{k}-\alpha_{Q}^{*}\right\|_{2}<\Delta$ and $\left\|x_{[Q]}^{k}-\mathbf{1}_{N} \tilde{x}_{Q}^{*}\right\|_{2}<\Delta$ for $k \geq k_{0}$. Then $\alpha_{Q}^{k}=\alpha_{Q}^{*}$ and $x_{[Q]}^{k}=\mathbf{1}_{N} \tilde{x}_{Q}^{*}$ due to the rounding quantization scheme. Therefore, $\left(x_{[Q]}^{k}, \alpha_{Q}^{k}\right)$ converges in finite iterations.

One may have noticed that the two terms in (27) converge relatively fast: $\psi^{k+1}\left(u_{Q}^{0}\right)$ is the $(k+1)$ th update of the ADMM and thus linearly convergent; $\sum_{i=0}^{k} u_{e^{\prime}}^{k-i}$ is absolutely bounded by the sum of two geometric series whose common ratios are both positive and less than 1. As such, we expect an upper bound for the number of iterations that guarantees the convergence of $\left(x_{[Q]}^{k}, \alpha_{Q}^{k}\right)$ to $\left(\mathbf{1}_{N} \tilde{x}_{Q}^{*}, \alpha_{Q}^{*}\right)$.

We first consider the number of iterations, denoted by $k_{1}$, that guarantees the convergence of $\alpha_{Q}^{k}$. Write $\beta_{Q}^{k}=$ $\beta_{I Q}^{k}+\beta_{E Q}^{k}$ where $\beta_{I Q}^{k}$ and $\beta_{E Q}^{k}$ are the corresponding vectors in the standard ADMM update $\psi^{k}\left(u_{Q}^{0}\right)$ and the 
accumulated error term $\sum_{i=0}^{k} u_{e^{\prime}}^{k-i}$ for $k \geq 1$, respectively. Define $u_{Q}^{k}=u_{I Q}^{k}+u_{E Q}^{k}$ analogously. Then we have

$$
\begin{aligned}
\left\|\alpha_{Q}^{k}-\alpha_{Q}^{*}\right\|_{2} & =\left\|M_{-}\left(\beta_{Q}^{k}-\beta_{Q}^{*}\right)\right\|_{2} \\
& =\left\|M_{-}\left(\beta_{I Q}^{k}-\beta_{I Q}^{*}+\beta_{E Q}^{k}-\beta_{E Q}^{*}\right)\right\|_{2} \\
& \leq \sigma_{\max }\left(M_{-}\right)\left(\left\|\beta_{I Q}^{k}-\beta_{I Q}^{*}\right\|_{2}+\left\|\beta_{E Q}^{k}-\beta_{E Q}^{*}\right\|_{2}\right) \\
& \leq \sqrt{\rho} \sigma_{\max }\left(M_{-}\right)\left(\left\|u_{I Q}^{k}-u^{*}\right\|_{G}+\lim _{\kappa \rightarrow \infty}\left\|\sum_{i=k+1}^{\kappa} u_{e^{\prime}}^{\kappa-i}\right\|_{G}\right),
\end{aligned}
$$

where the last inequality is from the definition of $G$. Since $\alpha_{Q}^{k}$ lies in the column space of $L_{-}$, we have from Lemma 4 that

$$
\left\|u_{I Q}^{k}-u^{*}\right\|_{G} \leq \frac{1}{(1+\eta)^{k}}\left\|u_{Q}^{0}-u^{*}\right\|_{G}
$$

Using the upper bound of (28), we get

$$
\begin{aligned}
\lim _{\kappa \rightarrow \infty}\left\|\sum_{i=k+1}^{\kappa} u_{e^{\prime}}^{\kappa-i}\right\|_{2} & \leq \lim _{\kappa \rightarrow \infty} \sum_{i=k+1}^{\kappa}\left\|u_{e^{\prime}}^{\kappa-i}\right\|_{2} \\
& \leq \sum_{i=k+1}^{\infty}\left(\frac{2}{(1+\eta)^{i+1}}\left\|u_{Q}^{0}-u^{*}\right\|_{G}+\frac{3}{(1+\eta)^{i}} \tau_{0}\right) \\
& \leq \sum_{i=k}^{\infty}\left(2\left\|u_{Q}^{0}-u^{*}\right\|_{G}+3 \tau_{0}\right) \frac{1}{(1+\eta)^{i}}
\end{aligned}
$$

Combining (34) and (35) yields

$$
\left\|u_{I Q}^{k}-u^{*}\right\|_{G}+\lim _{\kappa \rightarrow \infty}\left\|\sum_{i=k+1}^{\kappa} u_{e^{\prime}}^{\kappa-i}\right\|_{2} \leq 3\left(\left\|u_{Q}^{0}-u^{*}\right\|_{G}+\tau_{0}\right) \sum_{i=k}^{\infty} \frac{1}{(1+\eta)^{i}} .
$$

Hence, it suffices to pick $k_{1}$ such that

$$
3\left(\left\|u_{Q}^{0}-u^{*}\right\|_{G}+\tau_{0}\right) \sum_{i=k_{1}}^{\infty} \frac{1}{(1+\eta)^{i}}<\frac{\Delta}{\sqrt{\rho} \sigma_{\max }\left(M_{-}\right)},
$$

or,

$$
k_{1}=\left\lceil\log _{1+\eta} \frac{3 \sqrt{\rho} \sigma_{\max }\left(M_{-}\right)(1+\eta)\left(\left\|u_{Q}^{0}-u^{*}\right\|_{G}+\tau_{0}\right)}{\eta \Delta}\right\rceil .
$$

Though we have obtained $\alpha_{Q}^{k}=\alpha_{Q}^{*}$ for $k \geq k_{1}$, it is not enough to conclude $x_{[Q]}^{k_{1}}=\mathbf{1}_{N} \tilde{x}_{Q}^{*}$. We next use the fact that $x_{[Q]}^{k_{1}+1}$ reaches a consensus to find the number of iterations that guarantees the convergence of $x_{[Q]}^{k}$. Since $\alpha_{Q}^{k_{1}}$ has converged, we can write $x_{[Q]}^{k}=\mathbf{1}_{N} \zeta^{k}$ with $\zeta^{k} \in \Lambda^{M}$ for $k \geq k_{1}+1$. Thus $z_{Q}^{k}=\frac{1}{2} M_{+}^{T} x_{[Q]}^{k}=\mathbf{1}_{2 E} \zeta^{k}$ also reaches a consensus. Recalling that the $(k+1)$ th update of $[14 \mathrm{a})-(14 \mathrm{f})$ (which is properly initialized as discussed in Section $\amalg$ II-A is only based on $\left[z_{Q}^{k} ; \beta_{Q}^{k}\right]$, we only need to find $k_{2}$ such that $z_{Q}^{k}$ reaches $\mathbf{1}_{2 E} \tilde{x}_{Q}^{*}$ for $k \geq k_{2}$. Using 
the definitions of $u_{Q}^{k}$ and $G$, we get

$$
\begin{aligned}
\left\|z_{Q}^{k}-z_{Q}^{*}\right\|_{2} & \leq \frac{1}{\sqrt{\rho}}\left\|u_{Q}^{k}-u_{Q}^{*}\right\|_{G} \\
& \leq \frac{1}{\sqrt{\rho}}\left(\left\|u_{I Q}^{k}-u^{*}\right\|_{G}+\lim _{K_{1} \rightarrow \infty}\left\|\sum_{i=k+1}^{K_{1}} u_{e^{\prime}}^{K_{1}-i}\right\|_{G}\right) \\
& \stackrel{(a)}{\leq} \frac{3}{\sqrt{\rho}}\left(\left\|u_{Q}^{0}-u^{*}\right\|_{G}+\tau_{0}\right) \sum_{i=k}^{\infty} \frac{1}{(1+\eta)^{i}},
\end{aligned}
$$

where $(a)$ is from (36). Assume that $k_{2} \geq k_{1}+1$, i.e., $z_{Q}^{k}$ reaches a consensus for $k \geq k_{2}$. We can pick $k_{2}$ such that

$$
\left\|\zeta^{k_{2}}-\tilde{x}_{Q}^{*}\right\|=\frac{1}{\sqrt{2 E}}\left\|z_{Q}^{k_{2}}-z_{Q}^{*}\right\|_{2} \leq \frac{3}{\sqrt{2 \rho E}}\left(\left\|u_{Q}^{0}-u^{*}\right\|_{G}+\tau_{0}\right) \sum_{i=k_{2}}^{\infty} \frac{1}{(1+\eta)^{i}}<\Delta
$$

or

$$
k_{2}=\left\lceil\log _{1+\eta} \frac{3(1+\eta)\left(\left\|u_{Q}^{0}-u^{*}\right\|_{G}+\tau_{0}\right)}{\sqrt{2 \rho E \eta \Delta}}\right\rceil .
$$

If the above $k_{2}$ is less than $k_{1}+1$, then (37) must hold by picking $k_{2}=k_{1}+1$ since the consensus of $z_{Q}^{k}$ is reached for $k \geq k_{1}+1$.

In summary, the QC-ADMM converges within $\max \left\{k_{1}+1, k_{2}\right\}$ iterations.

Remark 1: We shall mention that the limit $\left(\mathbf{1}_{N} \tilde{x}_{Q}^{*}, \alpha_{Q}^{*}\right)$ need not be unique. This is because, unlike the standard ADMM, $\left\|u_{Q}^{k}-u^{*}\right\|_{G}$ in the QC-ADMM need not decrease monotonically due to the quantization that occurs on $x^{k}$ at each update. Note also that the given example illustrating the tightness of the consensus error bound is poorly initialized and we usually have smaller errors than the upper bound of (32) in practice (see simulations).

Remark 2: An interesting observation of the above theorem is the parameters $\mu$ given in Lemma 4 and $\rho$ which is the step size of the dual variable update. Without the knowledge of the network topology or the properties of local objective functions (namely, $m_{g}$ and $M_{g}$ defined in Lemma 4), one can hardly determine the optimal selection of these parameters under any criteria. In this sense, we do not regard $\mu$ and $\rho$ as a factor affecting the performance of the QC-ADMM, but may simply set, e.g., $\mu=\frac{3}{2}$ and $\rho=1$. Nevertheless, we will simulate the QC-ADMM with different $\rho$ for a distributed LASSO problem in Section IV.

Remark 3: To show the convergence of the QC-ADMM, our proof utilizes the linear convergence of the ADMM update $\psi$ on $u_{Q}^{k}$ and the boundedness of the error term $u_{e}^{k}$. As such, the main result for rounding quantization also holds for other deterministic quantizations as long as the quantization error is bounded. The proof idea also works for proving the convergence of other distributed algorithms with inexact updates: if the exactly updated variables converge relatively fast (e.g., $O\left(1 / k^{2}\right)$ and linear rate) and the error term on these variables at each update is deterministic and bounded, then this algorithm must converge. This idea will be used in Section $\amalg$ II-C to show the 
convergence results of the general objective functions that may contain the non-smooth components.

Remark 4: As previously mentioned, $x_{i}^{k}$ in the QC-ADMM is quantized for the $(k+1)$ th update at its own agent even though agents can compute and store real values with infinite precision. The reason is to guarantee that $\alpha_{Q}^{k}$ lies in the column space of $L_{-}$and that the QC-ADMM possesses the linear convergence rate at the ADMM update $\psi$ on $u_{Q}^{k}$ [cf. Equation (23)].

\section{Convergence Results: General Objective Functions}

We now investigate the general case where $f_{i}=g_{i}+h_{i}$. Even though (23) still represents the update of $u_{Q}^{k+1}$ from $u_{Q}^{k}$, the proof for smooth objective functions does not apply since the ADMM update $\psi$ no longer preserves the linear convergence property [cf. Equation (7)] due to the non-smooth component $h_{i}$. To proceed, we write explicitly $\partial f_{i}=\nabla g_{i}+\partial h_{i}$. Then the $x$-update (14a) is equivalent to

$$
\nabla g\left(x^{k+1}\right)+\partial h\left(x^{k+1}\right)+A^{T} \lambda_{Q}^{k}+\rho A^{T}\left(A x^{k+1}+B z_{Q}^{k}\right)=0 .
$$

We next make a further assumption on the smooth component $g_{i}$ :

Assumption 4: The solution to $\min _{\tilde{x}} \sum_{i=1}^{N} g_{i}(\tilde{x})$ is attainable.

This assumption together with Assumption 2 implies that there exists a unique $u^{\prime *}=\left[z^{\prime *} ; \beta^{\prime *}\right]$ which is the optimal value of solving $\min _{\tilde{x}} \sum_{i=1}^{N} g_{i}(\tilde{x})$ using the properly initialized ADMM. Next we consider the ADMM update on $u_{Q}^{k}=\left[z_{Q}^{k} ; \beta_{Q}^{k}\right]$ where only $g_{i}$ 's are involved, i.e.,

$$
\nabla g\left(x^{\prime k+1}\right)+A^{T} \lambda_{Q}^{k}+\rho A^{T}\left(A x^{\prime k+1}+B z_{Q}^{k}\right)=0,
$$

and the subsequent updates follow from (14b)-(14f). Here we use $x^{\prime k+1}, u^{\prime k+1}$, and $u_{Q}^{\prime k+1}$ to denote the corresponding updated values from (39) and (14b)-(14f) based on $u_{Q}^{k}=\left[z_{Q}^{k} ; \beta_{Q}^{k}\right]$. Denote by $\psi^{\prime}$ the update $u^{\prime k+1}$ from $u_{Q}^{k}$, i.e., $u^{\prime k+1}=\psi^{\prime}\left(u_{Q}^{k}\right)$. By Theorem 4, we have

$$
\left\|u^{\prime k+1}-u^{\prime *}\right\|_{G} \leq \frac{1}{1+\eta}\left\|u_{Q}^{k}-u^{\prime *}\right\|_{G}
$$

To apply the previous proof idea, we rewrite (23) as

$$
u_{Q}^{k+1}=\psi^{\prime}\left(u_{Q}^{k}\right)+\psi\left(u_{Q}^{k}\right)-\psi^{\prime}\left(u_{Q}^{k}\right)+u_{e}^{k}
$$

Treating $\psi\left(u_{Q}^{k}\right)-\psi^{\prime}\left(u_{Q}^{k}\right)+u_{e}^{k}$ as the error term, we see from Lemma 5 and Theorem 2 that the QC-ADMM must converge when $\psi\left(u_{Q}^{k}\right)-\psi^{\prime}\left(u_{Q}^{k}\right)+u_{e}^{k}$ is bounded throughout the updates. Note that $u_{e}^{k}$ is bounded as a result of 
the boundedness of $e^{k+1}$ [cf. Lemma 5], and that

$$
\psi\left(u_{Q}^{k}\right)-\psi^{\prime}\left(u_{Q}^{k}\right)=u^{k+1}-u^{\prime k+1}=\left[\begin{array}{c}
\frac{1}{2} M_{+}^{T} \\
\frac{1}{2} M_{-}^{T}
\end{array}\right]\left(x^{k+1}-x^{\prime k+1}\right) .
$$

The QC-ADMM thus converges as long as $\left(x^{k+1}-x^{\prime k+1}\right)$ is bounded, which is stated below.

Theorem 3: Consider the QC-ADMM algorithm. Suppose that Assumptions 14 hold. If $\left\|x^{\prime k+1}-x^{k+1}\right\|_{2} \leq \Delta_{x}$ for some $\Delta_{x}>0$ throughout the iterations, we have

1) Convergence: the sequence $\left(x_{[Q]}^{k}, \alpha_{Q}^{k}\right)$ generated by $[12)$ converges to a finite value $\left(\mathbf{1}_{N} \tilde{x}_{Q}^{*}, \alpha_{Q}^{*}\right)$ as $k \rightarrow \infty$, where $\tilde{x}_{Q}^{*} \in \Lambda^{M}$.

2) Consensus error: an upper bound for the consensus error is given by

$$
\left\|\tilde{x}_{Q}^{*}-\tilde{x}^{*}\right\|_{2} \leq\left(\frac{1}{2}+\rho \frac{2 E}{\sum_{i=1}^{N} m_{g_{i}}}\right) \sqrt{M} \Delta .
$$

3) Number of iterations: $\left(x_{[Q]}^{k}, \alpha_{Q}^{k}\right)$ converges within $\left\lceil\log _{1+\eta} \Omega\right\rceil$ iterations, where

$$
\tau_{1}=\left(\frac{1}{2} \Delta_{x}+\frac{1}{4} \Delta \sqrt{M}\right) \sqrt{\rho \sigma_{\max }^{2}\left(M_{+}\right)+\frac{1}{\rho} \sigma_{\max }^{2}\left(M_{-}\right)},
$$

and

$$
\Omega=\max \left\{\frac{3 \sqrt{\rho} \sigma_{\max }\left(M_{-}\right)(1+\eta)^{2}\left(\left\|u_{Q}^{0}-u^{\prime *}\right\|_{G}+\tau_{1}\right)}{\eta \Delta}, \frac{3(1+\eta)\left(\left\|u_{Q}^{0}-u^{\prime *}\right\|_{G}+\tau_{1}\right)}{\sqrt{2 \rho E} \eta \Delta}\right\} .
$$

Proof: We only outline the proof as it is similar to that of Theorem 2

Convergence: We first have

$$
\begin{aligned}
\left\|\psi\left(u_{Q}^{k}\right)-\psi^{\prime}\left(u_{Q}^{k}\right)+u_{e}^{k}\right\|_{G} & \leq\left\|\left[\begin{array}{c}
\frac{1}{2} M_{+}^{T} \\
\frac{1}{2} M_{-}^{T}
\end{array}\right]\left(x^{k+1}-x^{\prime k+1}\right)\right\|_{G}+\left\|u_{e}^{k}\right\|_{G} \\
& \stackrel{(a)}{\leq}\left(\frac{1}{2} \Delta_{x}+\frac{1}{4} \Delta \sqrt{M}\right) \sqrt{\rho \sigma_{\max }^{2}\left(M_{+}\right)+\frac{1}{\rho} \sigma_{\max }^{2}\left(M_{-}\right)} \\
& =\tau_{1},
\end{aligned}
$$

where $(a)$ is due to the definition of $G$-norm and (25). Then one can similarly obtain an upper bound on $u_{Q}^{k}$ which is

$$
\left\|u_{Q}^{k}\right\|_{G} \leq\left\|u_{Q}^{0}-u^{\prime *}\right\|_{G}+\left(1+\frac{1}{\eta}\right) \tau_{1}
$$

The rest of the proof is exactly the same as Theorem 2 
Consensus error: Replace $\nabla g_{i}$ with $\partial f_{i}$ in (30). Note that we again have $\sum_{i=1}^{N} \partial f_{i}\left(\tilde{x}^{*}\right)=0$, and (31) becomes

$$
\sum_{i=1}^{N}\left(\partial f_{i}\left(\tilde{x}_{Q}^{*}-e_{i}^{*}\right)-\partial f_{i}\left(\tilde{x}^{*}\right)\right)=\sum_{i=1}^{N} 2 \rho\left|\mathcal{N}_{i}\right| e_{i}^{*}
$$

Using the convexity and strong convexity assumptions, we know that

$$
\left(\partial f_{i}\left(\tilde{x}_{Q}^{*}-e_{i}^{*}\right)-\partial f_{i}\left(\tilde{x}^{*}\right)\right)^{T}\left(\tilde{x}_{Q}^{*}-e_{i}^{*}-\tilde{x}^{*}\right) \geq m_{g_{i}}\left\|\tilde{x}_{Q}^{*}-e_{i}^{*}-\tilde{x}^{*}\right\|_{2}^{2}
$$

and thus

$$
\left\|\partial f_{i}\left(\tilde{x}_{Q}^{*}-e_{i}^{*}\right)-\partial f_{i}\left(\tilde{x}^{*}\right)\right\|_{2} \geq m_{g_{i}}\left\|\tilde{x}_{Q}^{*}-e_{i}^{*}-\tilde{x}^{*}\right\|_{2}
$$

Then the upper bound (32) also holds.

Number of iterations: The bound for number of iterations can be obtained by replacing $u^{*}$ and $\tau_{0}$ with $u^{\prime *}$ and $\tau_{1}$, respectively.

We then provide two often used non-smooth functions that satisfy the condition $\left\|x^{\prime k+1}-x^{k+1}\right\|_{2} \leq \Delta_{x}: \ell_{1}$-norm and indicator function with bounded box set.

$\ell_{1}$-norm: Let $\|w\|_{1}$ denote the $\ell_{1}$-norm of a vector $w \in \mathbb{R}^{L}$. Then its subgradient is given by

$$
\left(\partial\|w\|_{1}\right)_{j}= \begin{cases}1, & w_{j}>0 \\ -1 \text { or } 1, & w_{j}=0 \\ -1, & w_{j}<0 .\end{cases}
$$

where $w_{j}$ is the $j$ th entry of $w$. Consider the non-smooth component $h_{i}(\tilde{x})=\xi_{i}\|\tilde{x}\|_{1}$ and hence $h(x)=\sum_{i=1}^{N} \xi_{i}\left\|x_{i}\right\|_{1}$, where $\xi_{i}>0$. Subtracting (39) from (38) we get

$$
\nabla g\left(x^{k+1}\right)+\rho A^{T} A x^{k+1}-\nabla g\left(x^{\prime k+1}\right)-\rho A^{T} A x^{\prime k+1}=-\partial h\left(x^{k+1}\right) .
$$

Since $A^{T} A=2 \rho W$, we have

$$
\begin{aligned}
& \left(\nabla g\left(x^{k+1}\right)-\nabla g\left(x^{\prime k+1}\right)\right)^{T}\left(x^{k+1}-x^{\prime k+1}\right)+\rho\left(A^{T} A\left(x^{k+1}-x^{\prime k+1}\right)\right)^{T}\left(x^{k+1}-x^{\prime k+1}\right) \\
\geq & \left(m_{g}+2 \rho|\mathcal{N}|_{\min }\right)\left\|x^{k+1}-x^{\prime k+1}\right\|_{2}^{2},
\end{aligned}
$$

where the last inequality is due to the strong convexity of $g_{i}$ and $|\mathcal{N}|_{\min }=\min _{i}\left|\mathcal{N}_{i}\right|$. Also, using the CauchySchwarz inequality we get

$$
\left(-\partial h\left(x^{k+1}\right)\right)^{T}\left(x^{k+1}-x^{\prime k+1}\right) \leq\|\partial\| x^{k+1}\left\|_{1}\right\|_{2}\left\|x^{k+1}-x^{\prime k+1}\right\|_{2} \leq\left(M \sum_{i=1}^{N} \xi_{i}^{2}\right)^{1 / 2}\left\|x^{k+1}-x^{\prime k+1}\right\|_{2} .
$$


Combining (43) and (44), we obtain that

$$
\left\|x^{k+1}-x^{\prime k+1}\right\|_{2} \leq \frac{\left(M \sum_{i=1}^{N} \xi_{i}^{2}\right)^{1 / 2}}{m_{g}+2 \rho|\mathcal{N}|_{\min }} .
$$

Indicator function with bounded box set: An indicator function is usually used when the optimization variable is subject to a constraint set. For example, if $w \in \mathcal{X} \subset \mathbb{R}^{L}$ for some set $\mathcal{X}$, then this can be included in the indicator function defined as

$$
I_{\mathcal{X}}(w)= \begin{cases}0, & \text { if } w \in \mathcal{X} \\ \infty, & \text { otherwise }\end{cases}
$$

We consider $f_{i}(\tilde{x})=g_{i}(\tilde{x})+I_{\mathcal{X}}(\tilde{x})$ where $\mathcal{X}$ is a nonempty compact box set, i.e., $\mathcal{X}=\left\{\tilde{x} \in \mathbb{R}^{M}: a \preceq \tilde{x} \preceq b\right\}$ with $a, b \in \mathbb{R}^{M}$ and $\preceq$ representing the component-wise inequality. From (12) it is clear that the $(k+1)$ th $x_{i}$-update at agent $i$ is equivalent to

$$
\begin{aligned}
x_{i}^{k+1} & =\underset{\tilde{x} \in \mathcal{X}}{\arg \min } g_{i}(\tilde{x})+\rho\left|\mathcal{N}_{i}\right| \tilde{x}^{T} \tilde{x}-\rho\left(\left|\mathcal{N}_{i}\right| x_{i[Q]}^{k}+\sum_{j \in \mathcal{N}_{i}} x_{j[Q]}^{k}\right)^{T} \tilde{x}+\left(\alpha_{i Q}^{k}\right)^{T} \tilde{x} \\
& =\underset{\tilde{x} \in \mathcal{X}}{\arg \min } G_{i}^{k}(\tilde{x})+\left(\alpha_{i Q}^{k}\right)^{T} \tilde{x},
\end{aligned}
$$

where we define $G_{i}^{k}(\tilde{x})=g_{i}(\tilde{x})+\rho\left|\mathcal{N}_{i}\right| \tilde{x}^{T} \tilde{x}-\rho\left(\left|\mathcal{N}_{i}\right| x_{i[Q]}^{k}+\sum_{j \in \mathcal{N}_{i}} x_{j[Q]}^{k}\right)^{T} \tilde{x}$ for ease of presentation. Define $Q_{0}=\sup \left\{\|\tilde{x}\|_{2}+\frac{1}{2} \Delta \sqrt{M} \mid \tilde{x} \in \mathcal{X}\right\}$. Then $\|\tilde{x}\|_{2} \leq Q_{0}$ and $\left\|\tilde{x}_{[Q]}\right\|_{2} \leq Q_{0}$ for any $\tilde{x} \in \mathcal{X}$. Since $g_{i}(\tilde{x})$ is convex and differentiable, there must exist $t_{i} \in \mathbb{R}$ such that $\left\|\nabla g_{i}(\tilde{x})\right\|_{2} \leq t_{i}$ for $\tilde{x} \in \mathcal{X}$. Noting that (46) implies $x_{i}^{k+1} \in \mathcal{X}$, one can easily verify that $G_{i}^{k+1}(\tilde{x})$ is Lipschitz continuous with parameter $t_{i}+4 \rho\left|\mathcal{N}_{i}\right| Q_{0}$. Denote the $l$ th entry of $x_{i}^{k}$ and $\alpha_{i Q}^{k}$ as $x_{i_{l}}^{k}$ and $\alpha_{i Q_{l}}^{k}$, respectively. We claim the following:

$$
x_{i_{l}}^{k+1}= \begin{cases}a_{i}, & \text { if } \alpha_{i Q_{l}}^{k}>t_{i}+4 \rho\left|\mathcal{N}_{i}\right| Q_{0}, \\ b_{i}, & \text { if } \alpha_{i Q_{l}}^{k}<-\left(t_{i}+4 \rho\left|\mathcal{N}_{i}\right| Q_{0}\right),\end{cases}
$$

for $k=1,2, \cdots$.

Assume that $x_{i_{l}}^{k+1}>a_{i}$ when $\alpha_{i Q_{l}}^{k}>t_{i}+4 \rho\left|\mathcal{N}_{i}\right| Q_{0}$. Let $\tilde{x}^{k+1} \in \mathbb{R}^{M}$ denote the vector with the $l$ th entry being $a_{i}-x_{i_{l}}^{k+1}$ and the rest entries being 0 . We have $x_{i}^{k+1}+\tilde{x}^{k+1} \in \mathcal{X}$, and

$$
\begin{aligned}
& G_{i}^{k}\left(x_{i}^{k+1}+\tilde{x}^{k+1}\right)+\left(\alpha_{i Q}^{k}\right)^{T}\left(x_{i}^{k+1}+\tilde{x}^{k+1}\right)-G_{i}^{k}\left(x_{i}^{k+1}\right)-\left(\alpha_{i Q}^{k}\right)^{T} x_{i}^{k+1} \\
\stackrel{(a)}{\leq} & \left(t_{i}+4 \rho\left|\mathcal{N}_{i}\right| Q_{0}\right)\left(x_{i_{l}}^{k+1}-a_{i}\right)-a_{i Q_{l}}^{k+1}\left(x_{i_{l}}^{k+1}-a_{i}\right) \\
= & \left(\alpha_{i Q_{l}}^{k+1}-\left(t_{i}+4 \rho\left|\mathcal{N}_{i}\right| Q_{0}\right)\right)\left(a_{i}-x_{i_{l}}^{k+1}\right),
\end{aligned}
$$


where $(a)$ is because $G_{i}^{k}$ is Lipschitz continuous for $k \geq 1$. Since $\alpha_{i Q_{1}}^{k+1}>t_{i}+4 \rho\left|\mathcal{N}_{i}\right| Q_{0}$ and $x_{i_{l}}^{k+1}>a_{i}$, (48) is negative, which contradicts the fact that $x_{i}^{k+1}$ minimizes $G_{i}^{k}(\tilde{x})+\left(\alpha_{i Q}^{k}\right)^{T} \tilde{x}$ over $\mathcal{X}$. That $x_{i_{l}}^{k+1}=b_{i}$ when $\alpha_{i Q_{l}}^{k}<-\left(t_{i}+4 \rho\left|\mathcal{N}_{i}\right| Q_{0}\right)$ can be similarly shown.

With 47 we demonstrate that for $k=0,1, \cdots$,

$$
\left\|\alpha_{i Q}^{k}\right\|_{2} \leq \max \left\{\left\|\alpha_{i Q}^{0}\right\|_{2}+2 \rho\left|\mathcal{N}_{i}\right| Q_{0},\left(t_{i}+6 \rho\left|\mathcal{N}_{i}\right| Q_{0}\right) \sqrt{M}\right\}
$$

Let $k \geq 1$. If $\alpha_{i Q_{l}}^{k}>t_{i}+4 \rho\left|\mathcal{N}_{i}\right| Q_{0}$, then $x_{i_{l}}^{k+1}=a_{i}$ as a result of (47). Since $x_{j}^{k+1} \in \mathcal{X}$ for $j \in \mathcal{N}_{i}$ and $x_{j_{l}}^{k+1} \geq a_{i}$, $\alpha_{i Q_{l}}^{k+1}=\alpha_{i Q_{l}}^{k}+\rho\left|\mathcal{N}_{i}\right| x_{i_{l}[Q]}^{k+1}-\rho \sum_{j \in \mathcal{N}_{i}} x_{j_{l}[Q]}^{k+1} \leq \alpha_{i Q_{l}}^{k}$. Similarly, $\alpha_{i Q_{l}}^{k+1} \geq \alpha_{i Q_{l}}^{k}$ if $\alpha_{i Q_{l}}^{k}<-\left(t_{i}+4 \rho\left|\mathcal{N}_{i}\right| Q_{0}\right)$. If $\left|\alpha_{i Q_{l}}^{k}\right| \leq t_{i}+4 \rho\left|\mathcal{N}_{i}\right| Q_{0}$, we have that $\left|\alpha_{i Q_{l}}^{k+1}\right| \leq\left|\alpha_{i Q_{l}}^{k}\right|+\rho\left|\mathcal{N}_{i}\right| x_{i_{l}[Q]}^{k+1}\left|+\rho \sum_{l \in \mathcal{N}_{i}}\right| x_{l_{j}[Q]}^{k+1}\left|\leq t_{i}+6 \rho\right| \mathcal{N}_{i} \mid Q_{0}$. When $k=0$, we obtain that $\left\|\alpha_{i Q}^{1}\right\|_{2}=\left\|\alpha_{i Q}^{0}+\rho\left|\mathcal{N}_{i}\right| x_{i[Q]}^{1}-\rho \sum_{j \in \mathcal{N}_{i}} x_{j[Q]}^{1}\right\|_{2} \leq\left\|\alpha_{i Q}^{0}\right\|_{2}+2 \rho\left|\mathcal{N}_{i}\right| Q_{0}$ as $x_{i}^{1} \in \mathcal{X}$ and $x_{j}^{1} \in \mathcal{X}$ for $j \in \mathcal{N}_{i}$. We finally derive (49) by the definition of the Euclidean norm.

Next we use the strong convexity of $g_{i}$ to show that $\left\|x^{\prime k+1}-x^{k+1}\right\|_{2}$ is bounded. Noting that $x_{i}^{\prime k+1}=$ $\arg \min _{\tilde{x}} G_{i}^{k}(\tilde{x})+\left(\alpha_{i Q}^{k}\right)^{T} \tilde{x}$, we get that $\nabla G_{i}^{k}\left(x_{i}^{\prime k+1}\right)+\alpha_{i Q}^{k}=0$. Thus,

$$
\begin{aligned}
\nabla G_{i}^{k}\left(x_{i}^{k+1}\right)+\alpha_{i Q}^{k} & =\nabla G_{i}\left(x_{i}^{k+1}\right)+\alpha_{i Q}^{k}-\nabla G_{i}^{k}\left(x_{i}^{\prime k+1}\right)-\alpha_{i Q}^{k} \\
& =\nabla g_{i}\left(x_{i}^{k+1}\right)+2 \rho\left|\mathcal{N}_{i}\right| x_{i}^{k+1}-\nabla g_{i}\left(x_{i}^{\prime k+1}\right)-2 \rho\left|\mathcal{N}_{i}\right| x_{i}^{\prime k+1}
\end{aligned}
$$

Since $g_{i}$ is strongly convex under Assumption 2, we have

$$
\left\|\nabla G_{i}^{k}\left(x_{i}^{k+1}\right)+\alpha_{i Q}^{k}\right\|_{2} \geq\left(m_{g_{i}}+2 \rho\left|\mathcal{N}_{i}\right|\right)\left\|x_{i}^{k+1}-x_{i}^{\prime k+1}\right\|_{2} .
$$

In addition, when $k \geq 1$,

$$
\begin{aligned}
\left\|\nabla G_{i}^{k}\left(x_{i}^{k+1}\right)+\alpha_{i Q}^{k}\right\|_{2} & =\left\|\nabla g_{i}\left(x_{i}^{k+1}\right)+2 \rho\left|\mathcal{N}_{i}\right| x_{i}^{k+1}-\rho\left(\left|\mathcal{N}_{i}\right| x_{i[Q]}^{k}+\sum_{j \in \mathcal{N}_{i}} x_{j[Q]}^{k}\right)+\alpha_{i Q}^{k}\right\|_{2} \\
& \leq t_{i}+4 \rho\left|\mathcal{N}_{i}\right| Q_{0}+\left\|\alpha_{i Q}^{k}\right\|_{2} .
\end{aligned}
$$

Combining (49), (50) and (51), we obtain that for $k \geq 1$,

$$
\left\|x_{i}^{k+1}-x_{i}^{\prime k+1}\right\|_{2} \leq \frac{\max \left\{\left\|\alpha_{i Q}^{0}\right\|_{2}+t_{i}+6 \rho\left|\mathcal{N}_{i}\right| Q_{0},(\sqrt{M}+1) t_{i}+(4+6 \sqrt{M}) \rho\left|\mathcal{N}_{i}\right| Q_{0}\right\}}{m_{g_{i}}+2 \rho\left|\mathcal{N}_{i}\right|},
$$

and hence

$$
\left\|x^{k+1}-x^{\prime k+1}\right\|_{2} \leq \sum_{i=1}^{N} \frac{\max \left\{\left\|\alpha_{i Q}^{0}\right\|_{2}+t_{i}+6 \rho\left|\mathcal{N}_{i}\right| Q_{0},(\sqrt{M}+1) t_{i}+(4+6 \sqrt{M}) \rho\left|\mathcal{N}_{i}\right| Q_{0}\right\}}{m_{g_{i}}+2 \rho\left|\mathcal{N}_{i}\right|}
$$

\section{Simulations}

In this section, we present some simulation results to examine previous theoretical analysis. 
To construct a connected network with $N$ nodes and $E$ edges, we generate a complete graph consisting of $N$ nodes, and then randomly remove $\frac{N(N-1)}{2}-E$ edges while ensuring that the network stays connected. We first consider the following distributed optimization problem:

$$
\begin{array}{ll}
\underset{\tilde{x}}{\operatorname{minimize}} & \sum_{i=1}^{N}\left|a_{i}\right|\|\tilde{x}\|_{2}^{2}+b_{i}^{T} \tilde{x} \\
\text { subject to } & 1_{M} \preceq \frac{\tilde{x}}{N} \preceq 1_{M},
\end{array}
$$

where $a_{i} \in \mathbb{R} \backslash\{0\}$ follows $\mathcal{N}(0,1)$ and $b_{i} \in \mathbb{R}^{M}$ has its entries following $\mathcal{N}\left(0, N^{4}\right)$. Since we do not assume a priori knowledge of the network structure, the quantized incremental algorithm does not work here. Also, the quantized subgradient method in [16] does not have results for constrained problems. Hence we can only use the QC-ADMM and the quantized dual averaging (Q-DA) method. Let $\Delta=1$ and $M=3$. Set $\rho=1$ and the initial variables $x_{i}^{0}=\alpha_{i Q}^{0}=0$ for the QC-ADMM. The proximal function is chosen as $e(x)=\frac{1}{2}\|x\|_{2}^{2}$ for the Q-DA. Fig. 1 shows the simulation results of a network with $N=40$ nodes where the maximum iterative error is defined by $\max _{i=1}^{N}\left\|x_{i[Q]}^{k}-\tilde{x}^{*}\right\|_{2}$.

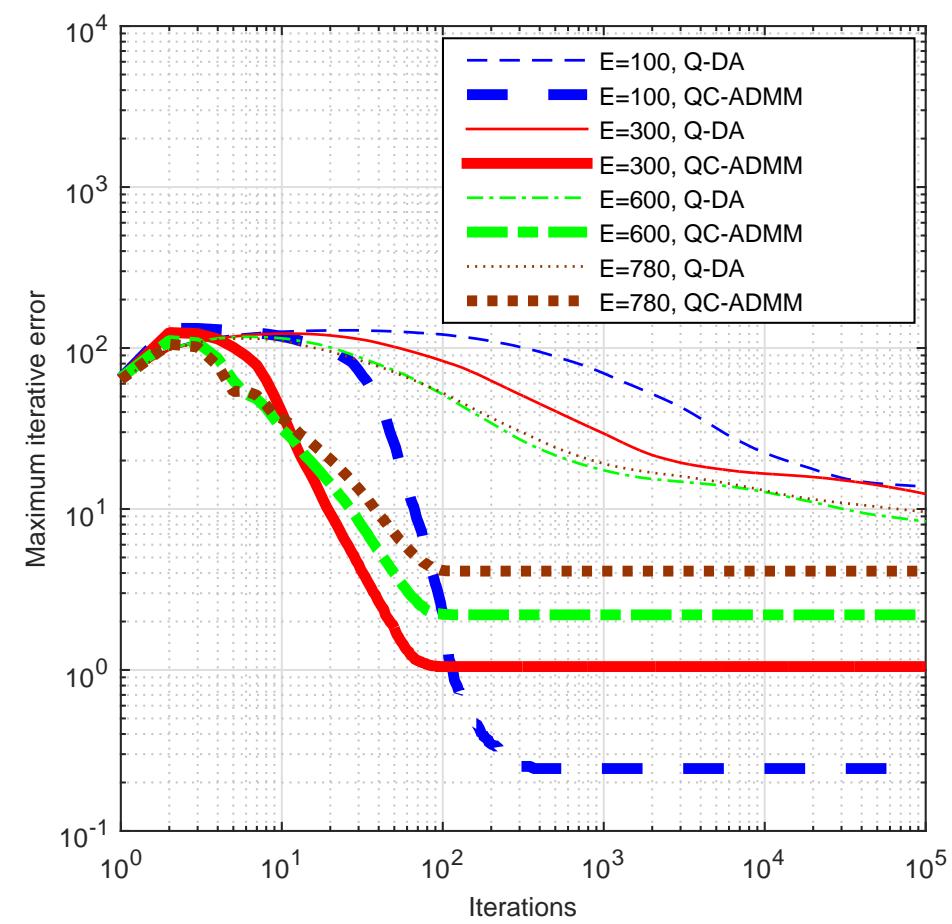

Fig. 1: Performance of the QC-ADMM and Q-DA where the plotted values are the average of 100 runs.

As seen from Fig. 1, the QC-ADMM has small maximum iterative errors and converges fast. Note that the QC-ADMM converges to a consensus in finite iterations, while the Q-DA only reaches a neighborhood of $\tilde{x}^{*}$ and does not guarantee the convergence or a consensus (see [15]). We also check that the practical consensus error of the QC-ADMM is usually much smaller than the upper bound in Theorem 3 For example, when $E=300$, 
the average upper bound in the simulation is 18.00 while the practical average consensus error is 1.05 . Another interesting observation is that when the graph becomes denser, i.e., $E$ becomes larger, the consensus error of the QC-ADMM tends to increase, which is in accordance with the upper bound for the consensus error.

We next consider a distributed LASSO problem:

$$
\underset{\tilde{x}}{\operatorname{minimize}} \sum_{i=1}^{N}\left\|A_{i} \tilde{x}-y_{i}\right\|_{2}+\lambda_{i}\|\tilde{x}\|_{1},
$$

where $A_{i} \in \mathbb{R}^{M \times M}$ is the linear measurement matrix of agent $i$ whose elements follow $\mathcal{N}(0,1), y_{i} \in \mathbb{R}^{M}$ is the measurement vector of agent $i$ whose elements follow $\mathcal{N}\left(0, N^{2}\right)$, and $\lambda_{i} \in \mathbb{R}^{+}$is a positive weight at agent $i$ and follows $\mathcal{N}\left(0, N^{2}\right)$. Let $M=20$ and $x_{i}^{0}=\alpha_{i Q}^{0}=0$. Define the iterative error as $\left\|x_{[Q]}^{k}-\mathbf{1}_{N} \tilde{x}^{*}\right\|_{2} / \sqrt{N}$ which is equal to the consensus error when a consensus is reached. In the following we study the effects of the quantization resolution, the algorithm parameter, and the graph density on the QC-ADMM via this LASSO problem.

Quantization resolution: Set $\rho=1$. Fig. 2 is the simulation result of a network with $N=40, E=300$ and $\Delta \in\{0,0.1,0.5,2.5,10\}$. Here $\Delta=0$ means that no quantization operation is placed on data communications, i.e., the C-ADMM is used to solve the LASSO problem.

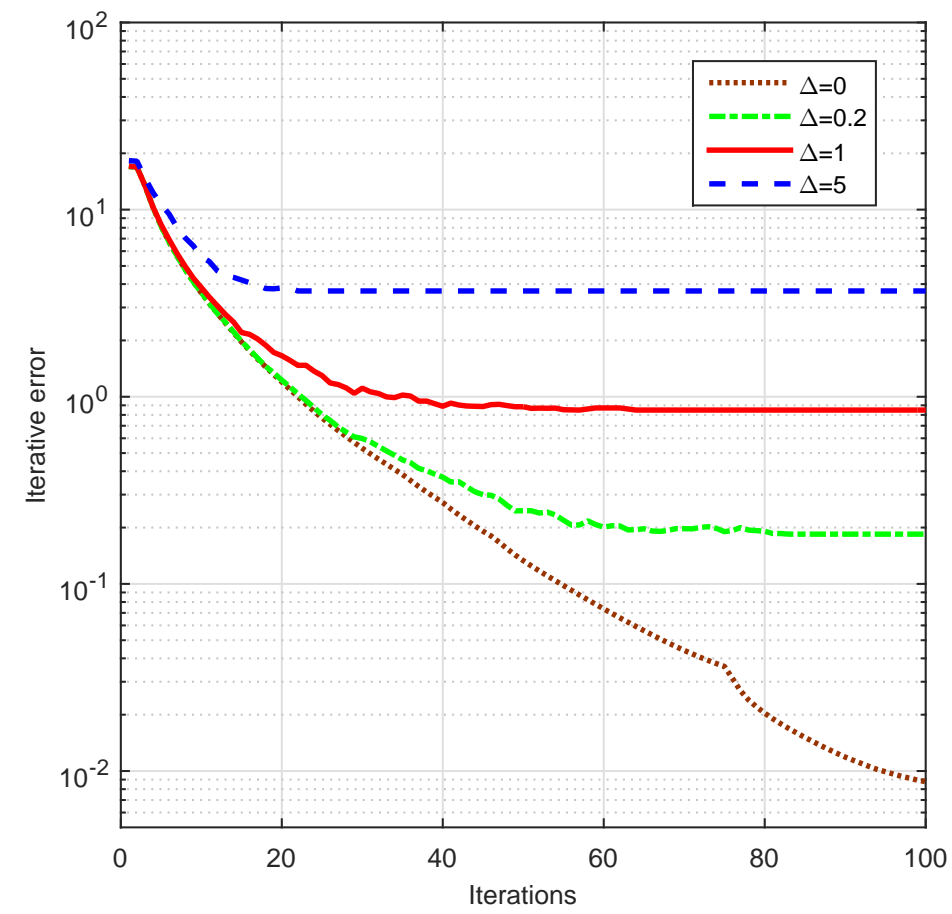

Fig. 2: The QC-ADMM for a distributed LASSO problem with $\rho=1, N=40, E=40$ and $\Delta \in\{0,0.1,0.5,2.5,10\}$.

We observe that the consensus error becomes larger as $\Delta$ increases. This is not surprising as the higher the quantization resolution is, the more information is lost at each update, thus resulting in a higher consensus error. Meanwhile, the convergence time decreases when $\Delta$ increases, which can be seen from the upper bound on the 
number of iterations that guarantees the convergence of the QC-ADMM; that is, $\Omega$ in (41) decreases as $\Delta$ becomes larger. On the other hand, a larger $\Delta$ indicates a sparser quantization lattice which makes it easier for the QC-ADMM reach a convergence point.

Algorithm parameter: From Theorems 2 and 3, the upper bound on the consensus error increases with the algorithm parameter $\rho$. However, characterizing the effect of $\rho$ on the convergence time is very hard: $\rho$ not only affects the linear convergence rate $\eta$, but also involves in the upper bound on the number of iterations that guarantees the convergence of the QC-ADMM. Moreover, as shown in [24], even though one can pick $\rho$ that maximizes the upper bound of $\eta$, the practical performance is usually suboptimal. Therefore, we only use a numerical example to study the effect of $\rho$ on the convergence time of the QC-ADMM.

Set $\Delta=1, N=40$ and $E=300$. We apply the QC-ADMM to solving the above Lasso problem with $\rho \in\{0.01,0.1,1,10\}$, and the result is presented in Fig. 3. In this example, the convergence time decreases as $\rho$ increases. Even though Theorem 3 indicates that a bigger $\rho$ results in a higher upper bound on the consensus error, the practical consensus error does not necessarily behave the same. That is, a bigger $\rho$ may lead a smaller consensus error in practice.

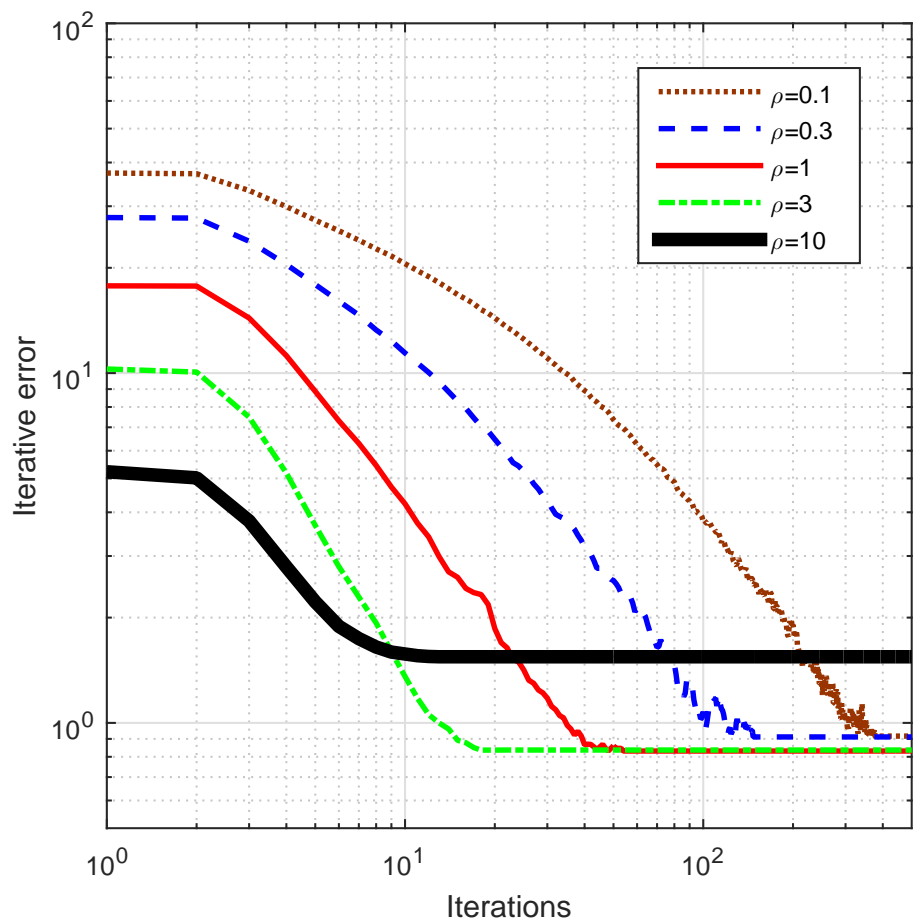

Fig. 3: The QC-ADMM for a distributed LASSO problem with $\Delta=1, N=40, E=40$ and $\rho \in\{0.01,0.1,1,10\}$.

Graph Density: Fig. 4 shows the result when $\Delta=1, \rho=1, N=40$, and $E \in\{100,300,500,780\}$. The consensus error is the same for all $E$ while the convergence time decreases as $E$ becomes larger. Again, though Theorem 3 indicates the upper bound for the consensus error increases in $E$ when $N$ is fixed, the practical consensus 
error need not necessarily perform the sam and can be much smaller. When $E$ increases with $N$ fixed, the average degree of the graph also increases. Then on the average, an agent can communicate with more agents at each update, thus resulting in a fast convergence.

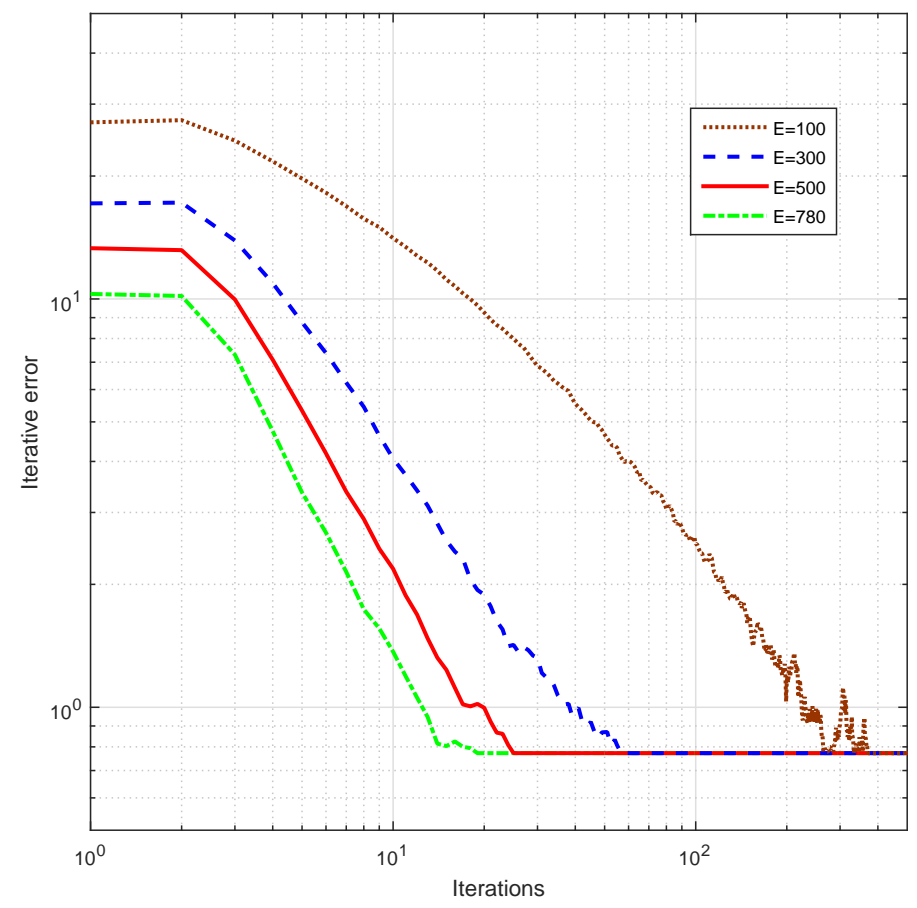

Fig. 4: The QC-ADMM for a distributed LASSO problem with $\Delta=1, \rho=1, N=40, E=\in\{100,300,500,780\}$.

\section{CONCLUSION}

This paper proposes an efficient algorithm, the QC-ADMM, for multi-agent distributed optimization under the quantized communication constraint. We show that this algorithm can be derived from the standard ADMM by adding a quantization operation on $x^{k}$ immediately after the $x$-update together with proper initializations. While existing quantized ADMM approaches only apply to quadratic local objectives, the QC-ADMM can deal with more general objective functions, possibly non-smooth. Specifically, the QC-ADMM converges to a consensus within finite iterations under certain convexity conditions, which further enables us to derive a tight upper bound on the consensus error. Moreover, the proof idea provides a framework for convergence proof of a class of inexact updated algorithms.

Our approach also motivates future research directions:

1) We assume the quantized data communication between agents to be perfect in this paper. In practice, channel impairment may lead to imperfect transmissions. Moreover, the links between agents may fail and the topology of the network may vary. It is thus interesting to investigate how our algorithm performs in such settings. 
2) Recent work of [30], [31] proposes computationally efficient distributed ADMM algorithms that have linear convergence rates under certain conditions. We expect that the idea of this paper can also lead to quantized ADMM algorithms with significantly reduced computational complexity.

\section{REFERENCES}

[1] D. Bertsekas and J. Tsitsiklis, Parallel and Distributed Computation: Numerical Methods, 2nd ed. Nashua, NH, USA: Athena Scientific, 1997.

[2] G. Giannakis, Q. Ling, G, Mateos, I. Schizas, and H. Zhu, "Decentralized learning for wireless communications and networking," ArXiv, 2015.

[3] W. Ren, R. W. Beard, and E. M. Atkins, "Information consensus in multivehicle cooperative control," IEEE Contr. Syst. Mag., vol. 27, no. 2, pp. 71-82, Apr. 2007.

[4] N. Lynch, Distributed Algorithms. San Francisco, CA: Morgan Kaufmann, 1996.

[5] L. Xiao, S. Boyd, and S. Lall, "A scheme for robust distributed sensor fusion based on average consensus," Proc. Int. Conf. Information Processing in Sensor Networks, Los Angeles, CA, Apr. 2005.

[6] R. Bekkerman, M. Bilenko, and J. Langford, Scaling Up Machine Learning: Parallel and Distributed Approaches. Cambridge University Press, 2012.

[7] G. R. Andrews, Foundations of Multithreaded, Parallel, and Distributed Programming. Addison-Wesley, 2007.

[8] H. Zhu, G. B. Giannakis, and A. Cano, "Distributed in-network channel decoding," IEEE Trans. Signal Process., vol. 57, no. 10, pp. 3970-3983, 2009.

[9] S. Zhu and B. Chen, "Quantized consensus by the ADMM: probabilistic versus deterministic quantizers," ArXiv, 2015.

[10] G. Mateos, J. Bazerque, and G. Giannakis, "Distributed sparse linear regression," IEEE Trans. Signal Process., vol. 58, no. 10, pp. 1856-1871, 2009.

[11] Q. Ling and Z. Tian, "Decentralized sparse signal recovery for compressive sleeping wireless sensor networks," IEEE Trans. Signal Process., vol. 58, no. 7, pp. 3816-3827, 2010.

[12] J. Bazerque and G. Giannakis, "Distributed spectrum sensing for cognitive radio networks by exploiting sparsity," IEEE Trans. Signal Process., vol. 58, no. 3, pp. 1847-1862, 2010.

[13] L. Gan, U. Topcu, and S. Low, "Optimal decentralized protocol for electric vehicle charging," IEEE Trans. Power Syst., vol. 28, no. 2, pp. 940-951, 2013.

[14] M. Rabbat and R. Nowark, "Quantized incremental algorithms for distributed optimization,” IEEE J. Sel. Areas Commun., vol. 23, no. 4, pp. 798-808, 2006.

[15] D. Yuan, S. Xu, H. Zhao, and L. Rong, "Distributed dual averaging method for multi-agent optimization with quantized communication," Syst. Control Lett., vol. 61, no. 11, pp. 1043-1061, 2012.

[16] A. Nedić, A. Olshevsky, A. Ozdaglar, and J. N. Tsitsiklis, "Distributed subgradient methods and quantization effects," in Proc. IEEE Conf. on Decision and Control, Cancun, Mexico, 2008.

[17] A. Nedić and D. P. Bertsekas, "Incremental subgradient methods for nondifferentiable optimization," SIAM J. Optim., vol. 56, no. 1, pp. 109-138, 2001.

[18] J. Duchi, A. Agarwal, and M. Wainwright, "Dual averaging for distributed optimization: convergence analysis and network scaling," IEEE Trans. Autom. Control, vol. 57, no.3, pp. 592-606, 2012.

[19] A. Nedić and A. Ozdaglar, "Distributed subgradient methods for multi-agent optimization," IEEE Trans. Autom. Control, vol. 54, no. 1, pp. 48-61, 2009. 
[20] S. Boyd, N. Parikh, E. Chu, B. Peleato, and J. Eckstein, "Distributed optimization and statistical learning via the alternating direction method of multipliers,” Foundat. Trends Mach. Learn., vol. 3, no. 1, pp. 1-122, 2010.

[21] B. He and X. Yuan, "On the $O(1 / n)$ convergence rate of the Douglas-Rachford alternating direction method," SIAM J. Numer. Anal., vol. 50, no. 2 pp. 700-709, 2012.

[22] M. Hong and Z.-Q. Luo, "On the linear convergence of the alternating direction method of multipliers," ArXiv, 2013.

[23] W. Deng and W. Yin, "On the global and linear convergence of the generalized alternating direction method of multipliers," Rice Univ. CAAM Tech Report, TR12-14, 2012.

[24] W. Shi, Q. Ling, K. Yuan, G. Wu, and W. Yin, "On the linear convergence of the ADMM in decentralized consensus optimization," IEEE Trans. Signal Process., vol. 62, no. 7, pp. 1750-1760, 2014.

[25] L. Schuchman, "Dither signals and their effect on quantization noise," IEEE Trans. Commun. Technol., vol. COMM-12, pp. 162-165, 1964.

[26] F. Chung, Spectral Graph Theory, Amer. Math. Soc., Providence, 1997.

[27] M. fiedler, “Algebra connectivity of graphs," Czechoslovake Math. J., vol. 23, no. 98, pp. 298-305, 1973.

[28] D. Cvetkovic, P. Rowlingson, and S. Simic "Signless Laplacians of finite graphs," Linear Algebra Appl., vol. 423, pp. 155-171, 2007.

[29] W. Rudin, Principles of Mathematical Analysis, New York: McGraw-Hill, 1976.

[30] T.-H. Chang, M. Hong, and X. Wang, "Multi-agent distributed optimization via inexact consensus ADMM," IEEE Trans. Signal Process., vol. 63, no. 2, pp. 482-497, 2015.

[31] Q. Ling and A. Ribeiro, "Decentralized linearized alternating direction method of multipliers," in Proc. IEEE Intl. Conf. on Acoustics, Speech, and Signal Process., Florence, Italy, 2014. 